Proc. Indlan Acad. Sci. (Earth Planet. Sci.), Vol, 90, No. 3, November 1981, pp. 305-326.

(C) Printed in India.

\title{
Envelope soliton solution for finite amplitude equatorial waves
}

\author{
R K JAIN*, B N GOSWAMI**, V SATYAN and \\ R N KESHAVAMURTY \\ Physical Research Laboratory, Ahmedabad 380 009, India \\ *Present address : Computer Centre, Sardar Patel University, \\ Vallabh Vidyanagar 388 120, India. \\ **Present address : Laboratory for Atmospheric Sciences, \\ Goddard space Flight Center, Greenbelt, MD 20770, USA.
}

MS received 26 December 1979 ; revised 22 July 1981

\begin{abstract}
Using shallow water equations on an equatorial beta plane, the nonlinear dynamics of the equatorial waves is investigated. A general mathematical procedure to study the nonlinear dynamics of these waves is developed using the asymptotic method of multiple scales. On faster temporal and spatial scales the equations describe the equatorial waves viz, the Rossby waves, Rossby gravity waves, the inertia gravity waves and the Kelvin waves. Assuming that the amplitude of these waves are functions of slower time and space scales, it is shown that the evolution of the amplitude of these waves is governed by the nonlinear Schrodinger equation. It is then shown that for the dispersive waves like Rossby waves and Rossby-gravity waves, the envelope of the amplitude of the waves has a 'soliton' structure.
\end{abstract}

Keywords. Shallow water equations ; fnite amplitude solutions ; multiple time scale method; envelope solitons.

\section{Introduction}

In the tropical and mid-latitude atmospheres, large (planetary) scale wave-like structures of the atmospheric flows are observed features of the general circulation and have been the subject of intense study among the theoretical and observational meteorologists. A large number of theoretical investigations on the linear stability analysis of atmospheric flows has been made with flows which are independent of time and longitude. Once the superimposed perturbations reach a finite amplitude or a fully developed eddy stage by deriving a substantial amount of energy from the zonal flow via either baroclinic or barotropic instability mechanisms or a combination of both, the total flow pattern becomes nonsteady and zonally non-uniform. Regardless of the energy 
sources of the atmospheric wave-like disturbances, their finite-amplitude existence is certain and therefore, nonlinear processes should play an important role in their dynamics.

For barotropic Rossby wave motion, instability has been established by Lorenz (1972). By employing linear perturbation analysis Lorenz showed that in the barotropic atmospheric flow, Rossby's (Rossby et al 1939) original solution (representing an east-west propagating planetary wave embedded in a constant westerly flow) is unstable provided the wave amplitude is sufficiently large or its wave number is suficiently high. It is clearly of interest to us to determine how the nature of the instability is affected by the nonlinear processes which become increasingly important as the perturbations superimposed upon the basic state reach a finite amplitude regime. A few questions of fundamental importance arise here:

(i) Given sufficient energy, how will the waves evolve in time and space? In other words, what is the structure and long time behaviour of the finite-amplitude waves?;

(ii) What are their propagation characteristics on slower time and space scales?;

(iii) How does the finite-amplitude perturbation state affect the basic flow in the long time?;

(iv) Are the fully developed zonally uniform or non-uniform and non-steady flows (basic zonal flow + finite amplitude waves) stable with respect to further perturbations. The present investigation addresses itself to these questions (or a few of them) which cannot be answered by a linear theory. The vast majority of theoretical investigations have been made to study the linear stability problem of unstable waves in the barotropic atmosphere on the equatorial and mid-latitude $\beta$-planes [Matsuno 1966; Longuet-Higgins and Gill 1967; Lorenz 1972; Duffy 1974 (to name a few) and references therein]. It was Phillips (1954) who first took account of some effects of nonlinearity and computed the second order changes in the basic current embedded in the unstable waves which exhibited exponential growth.

But this theory seems to have an unavoidable defect that each succeeding term in the amplitude expansion grows more rapidly than the previous term and therefore severely restricts the time for which the expansion remains valid. In recent past a number of nonlinear equations describing the evolution of finiteamplitude waves in dispersive media, have been shown to possess exact analytical solutions whose single most distinctive feature is the existence of solitary waves (solitons) [an excellent review on solitary solutions has been given by Scott et al (1973) ].

Investigations on the nonlinear time evolution of finite-amplitude waves in the baroclinic atmosphere, have been made by several research workers (Long 1964; Larsen 1965; Benney 1966; Pedlosky 1970, 1971, 1972a, b, 1977; Clarke 1971; Redekopp 1977; Redekopp and Weidmann 1978). But in the case of barotropic atmosphere with a zero basic zonal flow or a horizontally sheared zonal flow, only a few investigations have been made to study the nonlinear behaviour and time evolution of these waves (Gill 1974; Dommaracki 
and Loesch 1977; Loesch 1978; Boyd 1980). Only a few of these research workers have shown the existence of "solitary" solution for large scale planetary waves in both the middle latitude and equatorial atmospheres.

The present investigation represents an extension of the linear analyses of Matsuno (1966) into the nonlinear regime, while the perturbation is finite but small. Under this assumption, the nonlinear analysis may be carried out using asymptotic expansions for perturbations and their derivatives and using the procedure of multiple scales. In this paper we have studied the long time evolution of finite-amplitude (i.e. weakly nonlinear) and slowly varying wave train propagating in the atmosphere with a zero basic zonal flow, using the divergent barotropic model on a $\beta$-plane.

In $\S 2$, we have outlined our model and presented the governing equations and the formulation of the derivation of a modified nonlinear Schrodinger equation governing the amplitude modulation of the atmospheric waves. According to this equation, the waves have steady state "envelope soliton" solutions under certain conditions. Though dispersive waves in optics and in plasmas are known to have envelope soliton solutions, to our knowledge, this is a good theoretical evidence of the existence of 'envelope solitons' in any geophysical situation. We have also discussed briefly in this section the propagation characteristics of the linearized solutions [O(1) solutions] for these waves as the lowest order approximations of the model. In $\S 3$, we have obtained the envelope solitary wave structures (solitons) as the stationary solutions of the modified nonlinear Schrodinger equation. In $\$ 4$, we have discussed the results obtained. Finally, the results of our analysis are summarized in the concluding section.

\section{The model and the governing equations}

\subsection{Formulation}

The model we consider here, is the so called divergent barotropic model on a $\beta$ plane. We follow Matsuno's (1966) approach, retaining the nonlinear terms in the present analysis. The model consists of a single layer of homogeneous inviscid fluid of mean constant depth $H$, with a free surface under hydrostatic balance (figure 1). We take the local cartesian coordinate system shown in figure 1 , where $x^{\prime}$ is positive in the eastward direction. $y^{\prime}$ is taken in northward direction and $z^{\prime}$ is antiparallel to the gravity vector and is thus vertically upward.

We assume that the free surface deformations ' $h$ ' are sufficiently small $(h<H)$ so that these can be ignored when the effects of horizontal divergence aro incorporated into the model. We make here $\beta$-plane approximation whereby the important dynamic effects of the earth's rotation and sphericity on large scale motious are consicered by assuming the linear variation with latitude $\phi$ of the Coriolis parameter $f^{\prime}(=2 \Omega \sin \phi)$ as :

$$
f^{\prime}=f_{0}^{\prime}+\beta^{\prime} y^{\prime}
$$

where $y^{\prime}=a\left(\phi-\phi_{0}\right)$, i.e. the coordinate $y^{\prime}$ is centred at the latitude $\Phi_{0}, a$ is the 


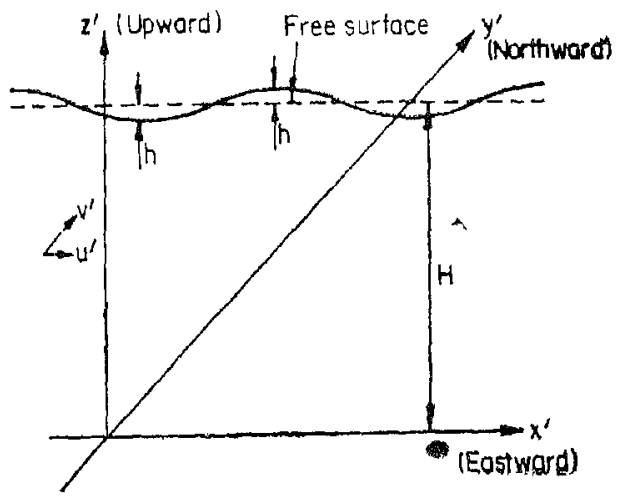

Figure 1. The model and the co-ordinate system.

mean earth radius, $f_{0}=2 \Omega_{0}$ sin $\phi_{0}(\Omega$ being the earth's angular velocity and $\beta^{\prime}\left[=\left(\partial f^{\prime} / \partial \partial \phi_{0} / a\right]\right.$ is the Rossby parameter taken as constant.

The governing equations and the appropriate boundary conditions for the model subject to the above approximations are

$$
\begin{aligned}
& \frac{\partial u^{\prime}}{\partial t^{\prime}}+u^{\prime} \frac{\partial u^{\prime}}{\partial x^{\prime}}+v^{\prime} \frac{\partial u^{\prime}}{\partial y^{\prime}}-\left(f_{0}{ }^{\prime}+\beta^{\prime} y^{\prime}\right) v^{\prime}+\frac{\partial \Phi^{\prime}}{\partial x^{\prime}}=0, \\
& \frac{\partial v^{\prime}}{\partial t}+u^{\prime} \frac{\partial v^{\prime}}{\partial x^{\prime}}+v^{\prime} \frac{\partial v^{\prime}}{\partial y^{\prime}}+\left(f_{0}^{\prime}+\beta^{\prime} y^{\prime}\right) u^{\prime}+\frac{\partial \Phi^{\prime}}{\partial y}=0, \\
& \frac{\partial \Phi^{\prime}}{\partial t}+u^{\prime} \frac{\partial \Phi^{\prime}}{\partial x^{\prime}}+v^{\prime} \frac{\partial \Phi^{\prime}}{\partial y^{\prime}}-g H\left(\frac{\partial u^{\prime}}{\partial x^{\prime}}+\frac{\partial v^{\prime}}{\partial y^{\prime}}\right)=0, \\
& u^{\prime} v^{\prime}, \Phi^{\prime} \rightarrow 0 \text { as } y^{\prime} \rightarrow \pm \infty
\end{aligned}
$$

where prime denotes the dimensional quantities; $u^{t}$ and $v^{\prime}$ are the components of horizontal velocity in zonal and meridional directions respectively; $\Phi^{t}$ is the geopotential of the free surface. Assuming that there is no averaged basic flow, $u^{\prime}, v^{\prime}$ and $\Phi^{\prime}$ here represent the perturbations around this state. Equation (4) is the integrated continuity equation assuming that $u^{\prime}$ and $v^{\prime}$ are independent of the vertical coordinate.

We now define non-dimensional variables as

$$
\begin{gathered}
(x, y)=\left(x^{\prime}, y^{\prime}\right) / L ;(u, v)=\left(u^{\prime}, v^{\prime}\right) / U \\
t=\left(\beta^{\prime} L\right) t^{\prime} ; \Phi=\Phi^{\prime} /\left(\beta^{\prime} L^{2} U\right),
\end{gathered}
$$

where $U$ is the horizontal characteristic velocity and $L$ is the horizontal characteristic length defined by $L^{2}=(g H)^{1 / 2} / \beta, g$ being acceleration due to gravity. The set of the governing equations (2) - (4) are transformed, using (6), into nondimensional form as 


$$
\begin{aligned}
& u_{t}+\epsilon\left(u u_{x}+v u_{y}\right)-\left(f_{0}+y\right) v+\Phi_{x}=0, \\
& v_{t}+\epsilon\left(u v_{x}+v v_{y}\right)+\left(f_{0}+y\right) u+\Phi_{y}=0, \\
& \phi_{t}+\epsilon\left(u \phi_{x}+x \Phi_{y}\right)+u_{x}+v_{y}=0,
\end{aligned}
$$

with the boundary conditions

$$
u, v, \phi \rightarrow 0 ; \text { as } y \rightarrow \pm \infty
$$

where $f_{0}=f_{0}^{\prime} / \beta^{\prime} L$ is the dimensionless planetary vorticity at the latitude $\phi_{0}$ and is thus a constant parameter. A subscript represents differentiation with respect to that variable: $E=U / \beta^{\prime} L^{2}$ is the equivalent Rossby number. For typical values of $U=10 \mathrm{~m} / \mathrm{sec}, H \simeq 10 \mathrm{~km}$ and $\epsilon \sim O\left(10^{-2}\right)$ i.e. $\epsilon$ is a small parameter much less than unity.

The equations $(7)-(9)$ are nonlinear in nature, with $\epsilon$ as a nondimensional smallness parameter representing a measure of the magnitude of the nonlinear products. We thus choose $\epsilon$ as an expansion parameter in the analysis developed here.

The derivative-expansion method suggests us to extend the independent variables $x$ and $t$ to the sets of independent variables

$$
X_{0}, X_{1}, X_{2}, \ldots \text { and } T_{0}, T_{1}, T_{2} \ldots \text {, }
$$

where long time and space scales (independent variables $x$ and $t$ ) are defined as

$$
X_{\mathrm{i}}=\epsilon^{\mathrm{i}} x ; T_{\mathrm{i}}=\epsilon^{\mathrm{i}} t, i=0,1,2,3, \ldots,
$$

which are incorporated in $(7)-(9)$ through the expansion of the derivative operators $\frac{\partial}{\partial x}$ ind $\frac{\partial}{\partial t}$ as

$$
\begin{aligned}
& \frac{\partial}{\partial t^{4}} \rightarrow \frac{\partial}{\partial T_{0}}+\epsilon \frac{\partial}{\partial T_{1}}+\epsilon^{2} \frac{\partial}{\partial T_{2}}+\ldots \\
& \frac{\partial}{\partial x} \rightarrow \frac{\partial}{\partial X_{0}}+\epsilon \frac{\partial}{\partial X_{1}}+{ }^{2} \epsilon \frac{\partial}{\partial X_{2}}+\ldots
\end{aligned}
$$

Accordingly, any dependent variable, a function of $(x, y, t)$, is now regarded as a function of $\left(X_{\mathrm{i}}, y, T_{\mathrm{i}}, i=0,1,2, \ldots\right)$. We assume further that the dependent variables, $u, v, \phi$ (perturbations from a uniform basic state under consideration) have the asymptotic expansions in $\epsilon$ of the following form :

$(u, v, \Phi)=\left(u^{(0)}, v^{(0)}, \Phi^{(0)}\right)+\epsilon\left(u^{(1)}, v^{(1)}, \Phi^{(1)}\right)+\epsilon^{2}\left(u^{(2)}, v^{(2)}, \Phi^{(2)}\right)+\ldots$

Substituting (13) and (14) into the governing equations (7) to (9) and equating coefficients of like powers of $\epsilon$, we obtain a set of perturbation equations to the different orders in $\epsilon$. These will then determine successively the perturbation quantities $\left(u^{(i)}, v^{(i)}, \Phi^{(i)} ; i=0,1,2 \ldots\right.$ to the different orders in $\epsilon$ as defined in (14). These dependent quantities are to be determined so as to be bounded 'non-secular) at each stage of perturbations. 


\subsection{O(1) problem : linear analysis}

To the $O(1)$, the perturbation equations determining $v^{(0)}, u^{(0)}$ and $\Phi^{(0)}$ and obtained by equating the coefficients of $\epsilon^{0}$ equal to zero are

$$
\begin{aligned}
& L v^{(0)}=0, \\
& P u^{(0)}-Q v^{(0)}=0, \\
& \Phi_{T 0}^{(0)}+u_{\mathrm{x} 0}^{0}+v_{Y}^{(0)}=0
\end{aligned}
$$

where $L \equiv \partial_{\mathrm{T} 0 \mathrm{ror} 0}-\partial_{\mathrm{x} 0 \mathrm{x} 0 \mathrm{x} 0}-\partial_{\mathrm{yy}} \mathrm{r} 0+y^{\mathrm{z}} \partial_{\mathrm{T} 0}-\partial_{\mathrm{x} 0}$

$$
P \equiv \partial \mathrm{T}_{0} \mathrm{~T}_{0}-\partial \mathrm{x}_{0} \mathrm{x}_{0} ; Q \equiv y \partial \mathrm{T} 0+\partial y x_{0}
$$

This set of equations (15) - (17) constitutes the linear problem for $O(1)$ solutions as nonlinear terms are absent.

As we are concerned with the problem of amplitude modulation, we assume the linearized problem to have a dispersive wave solution of the form $\exp \left[i\left(k x_{0}-\omega_{T 0}\right)\right]$, propagating in west-east direction, $k$ being the wave number and $w$ the frequency. Let the solution to (15) be

$$
v^{(0)}=\sum_{j}\left[A_{j}^{(0)}\left(X_{1}, T_{1}, \ldots\right) V_{j}^{(0)}(y) e^{i\left(k_{j} X_{0}-\omega_{j} T_{0}\right)}+c . c+\alpha_{j}\left(X_{1}, T_{1}, \ldots . .\right)\right.
$$

where $\mathrm{j}=0,1,2, \ldots$ and $A_{\mathrm{j}}^{(0)}$ are the complex wave amplitudes (constant with respect to $\left.X_{0}, T_{0}, y\right)$ which are assumed to be smooth slowly varying functions of slow time and space scales and satisfy $A_{j}^{(0)}\left(X_{1}, T_{1}, \ldots\right)=A_{j}^{(0) *}\left(-X_{1},-T_{1}, \ldots\right), \alpha_{1}$ are real constants, functions of higher order scales and can thus be termed as slow modes arising due to nonlinear interactions on slower time and space scales. Since we are concerned with the nonlinear modulation of the wave train represented by (18) and the nonlinear terms are absent in (15), we assume that $O$ (1) perturbation has the wave from which is devoid of the slower modes $\alpha_{j}$. Therefore, $\alpha_{j}=0$ is assumed here. If we take (18) as the $O(1)$ perturbation, we may treat interactions between the wave trains as slow modes. This case, however, is not treated in the present analysis.

Equations (15) to (17) are same as obtained by Matsuno (1966) who treated the $O(1)$ problem in detail and showed that the $O(1)$ problem is an eigen value problem. Following his approach, the orthogonal $O(1)$ eigen solutions belonging to eigenvalues $\omega_{j}$ can be obtained as (writing $\theta_{j}=k_{j} X_{0}-\omega_{j} T_{0}$ )

$$
\begin{aligned}
& v^{(0)}=\sum_{j}\left[-i\left(\omega_{j}^{2}-k_{j}^{2}\right) \psi_{n j}+c . c\right] \\
& u^{(0)}=\sum_{j}\left[\frac{1}{2}\left(\omega_{j}+k_{j}\right) \psi_{n j}+1\right. \\
& \left.\phi^{(0)}=\sum_{j}\left[\frac{1}{2}\left(\omega_{j}+k_{j}\right) \psi_{n_{j}+1}-n_{j}-k_{j}\right) \psi_{n_{j}-1}+c . c\right]
\end{aligned}
$$

where $\psi_{\mathrm{nj}} \not \neq A_{\mathrm{i}}^{(0)}\left(\mathrm{X}_{1}, \mathrm{~T}_{1}, \ldots\right)\left(\exp i \theta_{\mathrm{j}}=\exp _{\mathrm{j}}^{-}\left(-y^{2} / 2\right) H_{\mathrm{nj}}(y) ; n_{\mathrm{j}}=0,1,2 \ldots\right.$ 
and $H_{n:}$ is the Hermite polynomial of degree $n_{j}$ provided that the following dispersion relation is satisfied

$$
D\left(\omega_{\mathrm{j}}, k_{\mathrm{j}}\right) \equiv \omega_{\mathrm{j}}^{0}-\left(k_{\mathrm{j}}^{2}+2 n_{\mathrm{j}}+1\right) \omega_{\mathrm{j}}-k_{\mathrm{j}}=0 .
$$

Here $n_{1}$ can be indentified as meridional mode of solutions. This is the cubic dispersion relation whose roots define the characteristic wave types of this model. This gives a relationship between the frequency and the longitudinal wave number for some definite meridional mode $n_{j}$. For $n_{\mathrm{j}} \geqslant 1$ and using a trigonometric method of solution for cubic equation, the approximate values of three roots for $"$ i are given as

$$
\begin{aligned}
& \omega_{1 \mathrm{j}} \simeq\left(k_{\mathrm{j}}+2 n_{\mathrm{j}}+1\right)^{1 / 2}+{ }_{\mathrm{j}} k_{\mathrm{j}}\left(k_{\mathrm{j}}^{2}+2 n_{\mathrm{j}}+1\right)^{-1}-\frac{3}{8} k_{\mathrm{j}}^{2}\left(k_{\mathrm{j}}^{2}+2 n_{\mathrm{j}}+1\right)^{-5 / 2}, \\
& { }^{(\cdot)}{ }_{2 j}=-\left(k_{\mathrm{j}}+2 n^{\mathrm{j}}+1\right)^{1 / 2}+\frac{1}{2} k_{\mathrm{j}}\left(k_{\mathrm{j}}^{2}+2 n_{\mathrm{j}}+1\right)^{-1}+\frac{3}{3} k_{\mathrm{j}}^{2}\left(k_{\mathrm{j}}^{2}+2 n_{\mathrm{j}}+1\right)^{-5 / 2} \\
& w_{3 j} \simeq-k_{j}\left(k_{j}^{2}+2 n^{j}+1\right)^{-1}
\end{aligned}
$$

The upper two frequencies $\omega_{1}$ and $\omega_{2 j}$ correspond to respectively the frequencies of east and west propagating inertial-gravity waves, while $\omega_{3,}$, corresponds to the frequency of a westward propagating Rossby wave. The dispersion curves for these three frequencies for a few meridional modes $n_{\mathrm{j}}$ are shown in figure 2 . Fo $n_{\mathrm{j}} \geqslant 1$, these are completely separated from each other over the whole range of $k_{\mathrm{j}}$.

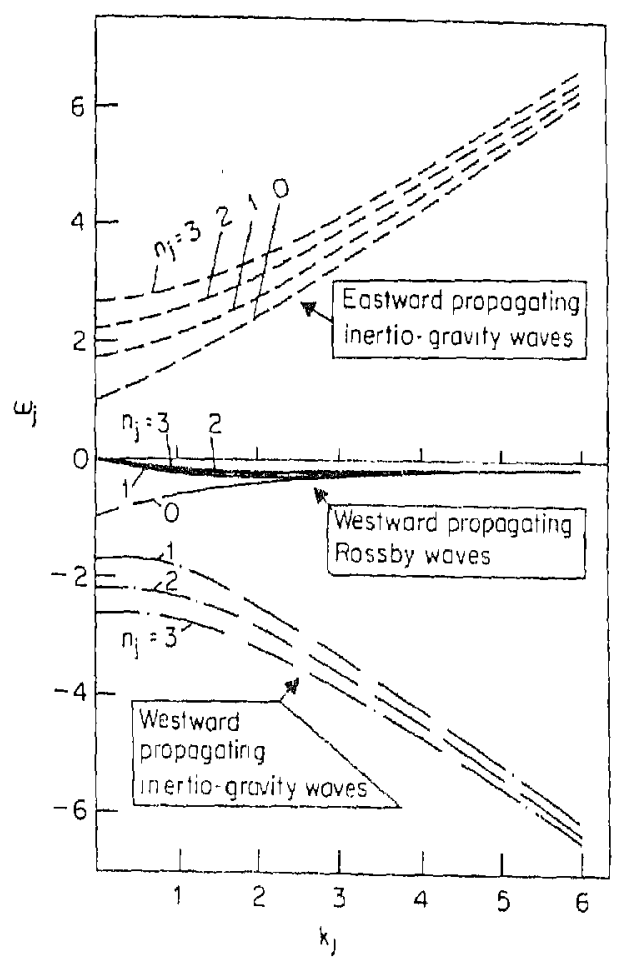

Figure 2. Frequencies as functions of wave number (dispersion curves). 
For the lowest mode $n^{l}=0$. The three roots of the dispersion relation (22) are

$$
\begin{aligned}
& \omega_{1 \mathrm{j}}=-k_{\mathrm{j}} \\
& \omega_{2 \mathrm{j}}=\frac{1}{2} k_{\mathrm{j}}+\left({ }_{4} k_{\mathrm{j}}^{2}+1\right)^{1 / 2} \\
& \omega_{3 \mathrm{j}}=\frac{1}{2} k_{\mathrm{j}}-\left(\frac{1}{4} k_{\mathrm{j}}^{2}+1\right)^{1 / 2}
\end{aligned}
$$

The first root $\omega_{1 \mathrm{j}}$ gives a trivial solution, $u^{(0)}=y^{(0)}=\Phi^{(0)}=0$ and hence, cannot be adopted as an eigenvalue of the $O(1)$ equations (15) - (17). Thus in the case of the lowest mode $n_{\mathrm{j}}=0$, we have only two waves, the frequency of one of which $\left(\omega_{2 j}\right)$ corresponds to an eastward propagating inertial-gravity wave and that of other $\left(\omega_{3 \mathrm{j}}\right)$ corresponds to a westward propagating mixed Rossby-gravity wave or Yanai wave. These are also shown in figure 2. As is obvious from figure 2, this mixed Rossby-gravity wave connects the two families of waves and its frequency ranges from a value which compared to the frequency of the inertial-gravity wave, to a value which is close to that of the Rossby wave.

\section{$2.3 O(\epsilon)$ problem: the finite-amplitude (non-linear stability analysis)}

To the $O(\epsilon)$, the solutions $v^{(1)}, u^{(1)}$ and $\Phi^{(1)}$ can be found out from the $O(\epsilon)$ perturbation equations which are obtained by equating the coefficient of $\epsilon$ equal to zero_and are

$$
\begin{aligned}
& L v^{(1)}=-L_{1} v^{(0)}-P\left(u^{(0)} v_{\mathrm{x} 0}^{(0)}+v^{(0)} v_{y}^{(0)}\right)-M\left(u^{(0)} u_{x 0}^{(0)}+v^{(0)} u_{y}^{(0)}\right)+N\left(u^{(0)} \Phi_{x 0}^{(0)}+v^{(0)} \Phi_{y}^{(0)}\right),(2) \\
& P u^{(1)}-Q v^{(1)}=-\left(P_{1} u^{(0)}-Q_{1} v^{(0)}\right)-\left(u^{(0)} u_{x 0}^{(0)}+v^{(0)} u_{y}^{(0)}\right)_{\mathrm{T} 0}-\left(u^{(0)} \Phi_{x 0}^{(0)}+v^{(0)} \Phi_{y}^{(0)}\right)_{x 0}
\end{aligned}
$$

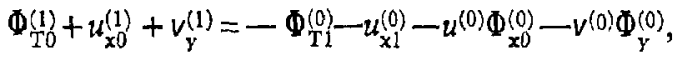

where $L_{1} \equiv 3 \partial_{\mathrm{ToT} 0 \mathrm{~T} 1}-\partial \mathrm{x}_{0 \mathrm{x} 0 \mathrm{~T} 1}-\partial_{\mathrm{yyT} 1}+y^{2} \partial_{\mathrm{T} 1}-\partial \mathrm{x}_{\mathrm{x}}-2 \partial_{\mathrm{x} 0 \mathrm{~T} 0 \mathrm{X} 1}$;

$$
\begin{aligned}
& P_{1} \equiv 2\left(\partial_{\mathrm{T} 0 \mathrm{~T} 1}-\partial \mathrm{x} 0 \mathrm{x} 1\right) ; Q_{1} \equiv y \partial \mathrm{T} 1+\partial_{\mathrm{yx} 1} ; \\
& M \equiv\left(\partial_{\mathrm{x} 0 \mathrm{y}}-y \partial_{\mathrm{T} 0}\right) ; N \equiv \partial_{\mathrm{yT} 0}-y \partial_{\mathrm{x} 0}
\end{aligned}
$$

It is obvious that the solutions $\nu^{(1)}, u^{(i)}$ and $\Phi^{(1)}$ of $(25)-(27)$ will be the linear combinations of the two solutions, namely (a) one solution of the homogeneous parts of (25) - (27) ; (b) another solution arising due to the inhomogeneity present on the r.h.s. of (25) - (27). As is clear from the above equations, knowing $v^{(1)}$ from (25) will enable us to solve for $u^{(1)}$ from $(26)$ and finally $\Phi^{(1)}$ from $(27)$. Since the operators appearing in 1.h.s. of $(25)-(27)$ are the same as those appearing in $(15)-(17)$ which determine $v^{(0)}, u^{(0)}$ and $\Phi^{(0)}$, the solutions of the homogeneous parts of $(25)-(27)$ (obtained by putting their r.h.s. $=0$ ) will be similar to the $O(1)$ solutions described by (19)-(21) with the dispersion relation (22) being satisfied, in their $\left(x_{0}, T_{0}, y\right)$ functional structures. Though the operators in 1.h.s. of (26) and (27) are the same as those in , 1.h.s. of $O(1)$ equations (16) and (17), the solutions of the homogeneous parts of these equations may be slightly different from $O(1)$ solutions because these operators now operate on $v^{(1)}$ 
and $u^{(1)}$ as well. It can be noticed from the linear inhomogeneous terms of (25) - (27) thit for every component of $V^{(1)} \Phi^{(1)}$ and $u^{(1)}$ there exists a corresponding resonant forcing arising from the introduction of multiple scales $\left(T_{1}, X_{1}\right)$ which are involved in the operators $L_{1}, P_{1}$ and $Q_{1}$. Nonlinear inhomogeneous terms may also give rise to resonant interactions under special conditions. Both linear and inhomogeneous resonant forcings will produce higher harmonics in the fundamental wave on $O(E)$ space and time scales, $X_{1}$ and $T_{\mid}$. Thus, considering that on these scales the second harmonics are produced due to resonant forcings, we assume the $O(\epsilon)$ solutions of the following forms :

$$
\begin{aligned}
& v^{(1)}=\sum_{1}\left[-i\left(\omega_{\mathrm{j}}^{2}-k_{\mathrm{j}}^{2}\right) \psi_{\mathrm{nj}}^{(1)}+B_{\mathrm{lj}}^{(1)}\left(X_{1}, T_{1}, \ldots\right) \exp 2 i \theta_{\mathrm{j}} \gamma_{\mathrm{rij}}^{(1)}(y)+c \cdot c+c_{1 \mathrm{j}}\right] \\
& u^{(1)} \sum_{j}\left[A_{1 j}^{(1)}\left(X_{1}, T_{1}, \ldots\right) \exp i \theta_{j} W_{1 j}^{(1)}(y)+B_{i j}^{(1)}\left(X_{1}, T_{1}, \ldots\right)\right. \\
& \exp \left(2 i \theta_{\mathrm{i}}\right) W_{2 \mathrm{j}}^{(1)}(y)+c \cdot c+c_{2 \mathrm{j}} \mathrm{j} \\
& \phi^{(1)}=\sum_{j}\left[A_{2 j}^{(1)}\left(X_{1}, T_{1} \ldots\right) \exp i \theta_{j} Z_{1 j}^{(1,}(y)+B_{3 j}^{(1)}\left(X_{1}, T_{1}, \ldots\right)\right. \\
& \left.\exp (2 i \theta \mathrm{j}) Z_{\mathrm{z}^{j}}^{(1)}(y)+c . c+c_{3 \mathrm{j}}\right]
\end{aligned}
$$

where $\psi_{\mathrm{ni}}^{(1)} \cdot A_{\mathrm{j}}^{(1)}\left(X_{1}, T_{1}, \ldots\right) \exp \left(i \Theta^{\mathrm{j}}\right) \exp \left(y^{2} / 2\right) H_{\mathrm{nj}}^{\mathrm{r}}(\mathrm{y})$. Since we are interested in the nonlinear modulation of the amplitude $A_{j}^{(0)}$ of the wave train (described by the $O(1)$ solutions), we assume that $A_{\mathrm{j}}^{(1)}, A_{(1,2) \mathrm{j}}^{(1)}$ and $B_{(1,2,3 \mathrm{j})}^{(1)}$ are functions of $A_{\mathrm{j}}^{(0)}$ and $A_{\mathrm{j}}^{(0) *}$ (asterisk denoting the complex conjugate) and thus are complex constants with respect to $X_{0}, T_{0}$ and $y . C_{(1,2,3) \mathrm{j}}$ are real constants with respect to $X_{0}, T_{0}$ and $y$ and assumed to be functions of $A_{j}^{(0)}, A_{j}^{(0) *}$. These constants are to be determined from the secularity removal conditions for higher order terms in $\epsilon . \quad V_{\mathrm{nij}}^{(1)}, W_{(1,2) \mathrm{j},}^{(1)}, Z_{(1,2) \mathrm{j}}^{(1) !}$ are functions of $y$, yet to be determined.

Substitution of (28) into (25) will give us an equation which will determine the solution $v^{(1)}$ completely. The structure of $v^{(1)}$ so obtained would contain resonant secular terms (proportional to $\exp \left( \pm i \theta_{j}\right)$ unless the coefficients of $\exp \left( \pm i \theta_{j}\right)$ vanish. Equating the coefficients of $\exp \left(2 i \theta_{j}\right)$ in both sides of this equation will determine $B_{1 j}^{(1)}$ and $V_{r . j}^{(1)}$. In order that the solution for $V^{(1)}$ be non-secular, we must have

$$
A_{\mathrm{ij} T 1}^{(0)}+V_{\mathrm{B}} A_{\mathrm{j} \times 1}^{(0)}=0,
$$

which is the condition for non-secularity and thus removes the secular behaviour of $V^{(1)}$. By considering the complex conjugate terms, we shall get an exactly similar non-secular condition for $A_{j}^{(0)^{*}}$. Here $V_{g}$ is the group velocity of the waves defined by

$$
V_{\mathrm{g}}=-D_{\mathrm{kj}} / D \omega_{\mathrm{j}}=\left(1+2 \omega^{\prime} k_{\mathrm{j}}\right)\left(3 \omega_{\mathrm{j}}^{2}-k_{\mathrm{j}}^{2}-2 n-1\right)^{-1}
$$

$D$ being the dispersion function defined by (22). Under these non-secular conditions (3i) and equating the coefficients of $\exp \left( \pm 2 i \theta_{j}\right)$, we obtain the secularfree solution $\nu^{(1)}$ as

$$
v^{\prime}()=\sum_{j}\left[-i\left(\omega_{j}^{2}-k_{\mathrm{j}}^{2}\right) \psi_{(\mathrm{nj}}^{(i)}+\frac{i}{2\left(u_{j} j\right.} \psi_{\mathrm{nj}}^{(1)}+c \cdot c+c_{\mathrm{ij}}\right],
$$


where $\psi_{n j}^{(1)}=K A_{\mathrm{j}}^{(0) 2} \exp \left(2 i \theta_{\mathrm{j}}\right) V_{\mathrm{nj}}^{(i)}(y), K$ being the constant of proportionality and $V_{\mathrm{nj}}^{(l)}$ is determined by solving the following differential equation

$$
\frac{d^{2} V_{\mathrm{ni}}^{(1)}}{d y^{2}}+\left(4()_{\mathrm{j}}^{2}-4 k_{\mathrm{j}}^{2}-k_{\mathrm{j}} / \omega_{\mathrm{j}}-y^{2}\right) V_{\mathrm{nj}}^{(1)}=K^{-1} F\left({ }^{\prime} \mathrm{j}_{1} \cdot k_{\mathrm{j},} y\right),
$$

where we have replaced the partial derivative $\partial^{2} / \partial y^{2}$ by the total derivation $d^{2} / d y^{2}$. The function $F\left(n, j, k_{j}, y\right)$ appearing in (34) is given by

$$
F\left(\omega_{\mathrm{j}}, k_{\mathrm{j}}, y\right)=\left[F_{1 \mathrm{i}} H_{\mathrm{nj}}^{2}+F_{2 \mathrm{i}} H_{\mathrm{nj}} H_{\mathrm{nj}+1}+F_{3,} H_{\mathrm{nj}+1}^{2}\right] \exp \left(-\mathrm{y}^{2}\right)
$$

where $F_{(1,2,3, j}$ are all functions of $\mathrm{i}_{\mathrm{j}}, k_{\mathrm{j}}$ and $y$ and are given by

$$
\begin{aligned}
& F_{1 \mathrm{j}}\left(\omega_{\mathrm{j}}, k_{\mathrm{j}}, y\right)=2\left[y^{3}\left(\omega_{\mathrm{j}}-k_{\mathrm{j}}\right)^{2}\left(2 \omega_{\mathrm{j}}^{2}-k_{\mathrm{j}}^{2}\right)+2 y^{2} k_{\mathrm{j}}^{2}\left(\omega_{\mathrm{j}}-k_{\mathrm{j}}\right)^{2}-y\left(\omega_{\mathrm{j}}-k_{\mathrm{j}}\right)\right. \\
& \left\{2 \omega_{\mathrm{j}}\left(\omega_{\mathrm{j}}^{2}-k_{\mathrm{j}}^{2}\right)\left(\omega_{\mathrm{j}}^{2}-k_{\mathrm{j}}^{2}+\frac{1}{2}\right)+\left(\omega_{\mathrm{j}}+k_{\mathrm{j}}\right)\left(4 n_{\mathrm{j}} \omega_{\mathrm{j}}^{2}+4 k_{\mathrm{j}} \omega_{\mathrm{j}}-k_{\mathrm{j}}^{2}\right)\right. \\
& \left.-k_{\mathrm{j}} \omega_{\mathrm{j}}\left(n_{\mathrm{j}}+1\right)\left(\omega_{\mathrm{j}}-k_{\mathrm{j}}\right)\right\}+\left(\omega_{\mathrm{j}}-k_{\mathrm{j}}\right)\left\{\omega_{\mathrm{j}}\left(\omega_{\mathrm{j}}+k_{\mathrm{j}}\right)^{2}+2 k_{\mathrm{j}}\left(\omega_{\mathrm{j}}^{2}-k_{1}^{2}\right)\right. \\
& \left.\left.\quad+4 n_{\mathrm{j}} \omega_{\mathrm{j}} k_{\mathrm{j}}^{2}\right\}\right] \\
& F_{2 \mathrm{j}}\left(\omega_{\mathrm{j}}, k_{\mathrm{j}}, y\right)=2\left[y^{2}\left(\omega_{\mathrm{j}}-k_{\mathrm{j}}\right)\left\{\left(\omega_{\mathrm{j}}-k_{\mathrm{j}}\right)^{3}-\omega_{\mathrm{j}} k_{\mathrm{j}}\left(\omega_{\mathrm{j}}-5 k_{\mathrm{j}}\right)\right\} U-\right. \\
& y\left\{k_{\mathrm{j}}^{2}\left(\omega_{\mathrm{j}}^{2}+k_{\mathrm{j}}^{2}\right)-2 \omega_{\mathrm{j}} k_{\mathrm{j}}\right\}-2\left(\omega_{\mathrm{j}}^{2}-k_{\mathrm{j}}^{2}\right)^{2}\left(\omega_{\mathrm{j}}^{2}-1\right)-\frac{1}{4}\left(\omega_{\mathrm{j}}+k_{\mathrm{j}}\right) \\
& \quad\left\{\left(\omega_{\mathrm{j}}-k_{\mathrm{j}}\right)\left(6 \omega_{\mathrm{j}} \mathrm{j}+5 k^{\mathrm{j}} \omega_{\mathrm{j}}\right)+2 k_{\mathrm{j}}\left(\omega_{\mathrm{j}}^{2}-k_{\mathrm{j}}^{2}\right)\right\}+i 2 n_{\mathrm{j}}\left\{2\left(\omega_{\mathrm{j}}^{2}-k_{\mathrm{j}}^{2}\right)^{2}\right. \\
& \left.\left.\quad+k_{\mathrm{j}}^{3}\right\}\right], \\
& F_{3 \mathrm{j}}\left(\omega_{\mathrm{j}}, k_{\mathrm{j}}, y\right)=y_{\mathrm{j}}\left\{2\left(\omega_{\mathrm{j}}^{2}-k_{\mathrm{j}}^{2}\right)+k_{\mathrm{j}}\left(3 \omega_{\mathrm{j}}^{2}+k_{\mathrm{j}}^{2}\right\}-2 \omega_{\mathrm{j}} k_{\mathrm{j}}^{2} .\right.
\end{aligned}
$$

The secularity removal condition (31) essentially means that to the $O(E)$, the amplitude $A_{j}^{(0)}$ is constant in a frame of reference moving with the group velocity $V_{5}$. In other words, $A_{j}^{(0)}$ depends on $X_{1}$ and $T_{1}$ only through $\xi=X_{1}-V_{\mathrm{g}} T_{1}$.

The $y$-structure of the $O(\epsilon)$ perturbation in $v$ is governed by (34) which is an inhomogeneous, second-order ordinary differential equation. Using power series and green's function methods (Margenau and Murphy 1966) the solution of (34) can be formally written as

$$
V_{\mathrm{n} j}^{(1)}=\frac{1}{k} \int G(\delta, y) F\left(\omega_{\mathrm{j}}, k^{j}, \delta\right) d \delta,
$$

satisfying the boundary conditions. $\delta$ is a point which divides the range of $y$ from $-L_{y}$ to $L_{\mathrm{y}}$ into two parts (i) $-L_{\mathrm{y}}<y<\delta$ and (ii) $\delta<y<L_{\mathrm{y}} \cdot G(\delta, y)$ is the Green's function for the homogeneous differential equation (34), with boundary conditions same as those for $V_{\mathrm{a} j}^{(1)}$ The integrals in (37) can be evaluated analytically or numerically so that $V_{n j}^{(1)}$ is known. The structure of $V_{\mathbf{n}}^{(1)}$ for a special case corresponding to $n_{\mathrm{j}}=1$ is shown in figure 3 . Once $V_{\mathrm{nj}}^{(1)}$ is known $V^{(1)}$ described by (33) is determined. 


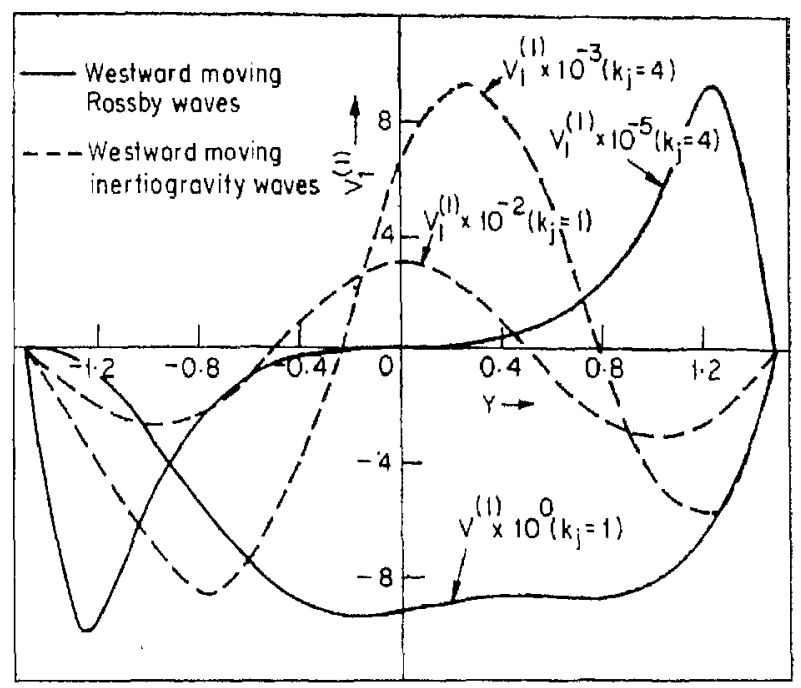

Figure 3. $V_{n j}(1)$ as functions of $Y\left(=f_{0}+y\right)$ for the meridional mode $n j=1$.

Now substituting $v^{(1)}$ and $u^{(1)}$ represented by (29) into (26) which governs $u^{(1)}$, subsequently removing the secular terms (i.e., equating the coefficients of $\exp \left( \pm i \theta_{\mathrm{j}}\right)$ to zero) which will determine $A_{\mathrm{ij}}^{(1)}, W_{1 \mathrm{j}}^{(1)}$ (and its c. c. equivalent) and equating the coefficients of exp! $\left(. t 2 i \theta_{j}\right)$ which will determine $B_{2 j}^{(1)} W_{2 j}^{(1)}$ (and its c.c. equivalent), we get the secular-free solution $u^{(1)}$ in terms of $V_{\mathrm{nj}}^{(1)}$ as

$$
\begin{aligned}
& u^{(1)}=\sum_{j}\left[y\left(\omega_{\mathrm{j}}-k_{\mathrm{j}}\right) \psi_{\mathrm{nj}}^{(1)}+k_{\mathrm{j}} \psi_{\mathrm{nj}+1}^{(1)}-i\left(\omega_{\mathrm{j}}^{2}-k_{\mathrm{j}}^{2}\right)^{-1}\left\{\left[y\left(\omega_{\mathrm{j}}^{2}-k_{\mathrm{j}}^{2}\right) \psi_{\mathrm{nj}}\right.\right.\right. \\
& \left.-2 \omega_{\mathrm{j}} k_{\mathrm{j}} \psi_{\mathrm{nj}+1}\right] A_{\mathrm{j} T 1}^{(0)} / A_{\mathrm{j}}^{(0)}-\left[y\left(\omega_{\mathrm{i}}^{2}-k_{\mathrm{j}}^{2}\right) \psi_{\mathrm{nj}}+\left(\omega_{\mathrm{j}}^{2}-k_{\mathrm{j}}^{2}\right) \psi_{\mathrm{nj}+1}\right] \times \\
& \left.A_{\mathrm{j} \times 1}^{(0)} / A_{\mathrm{j}}^{(0)}\right\}+\frac{1}{2}\left(\omega_{\mathrm{j}}^{2}-k_{\mathrm{j}}^{2}\right)^{-1}\left\{y \psi_{\mathrm{nj}}^{(1)}+\left(k_{\mathrm{j}} / \omega_{\mathrm{j}}\right) \psi_{\mathrm{nj}}^{(1)} V_{\mathrm{nj}}^{(1)} / V_{\mathrm{nj}}^{(1)}\right\} \\
& -\frac{1}{2}\left\{\left[y^{2}\left(\left(\omega_{\mathrm{j}}^{2}-k_{\mathrm{j}}^{2}\right) \omega_{\mathrm{j}}+\left(\omega_{\mathrm{j}}^{2}-k_{\mathrm{j}}^{2}\right)\right] \psi_{\mathrm{nj}}^{2}-y \omega_{\mathrm{j}}^{2} \psi_{\mathrm{nj}} \psi_{\mathrm{nj}+1}\right\}\right. \\
& \left.+c . c+c_{2 \mathrm{j}}\right] .
\end{aligned}
$$

Sinfilarly, the solution of (27) which governs $\Phi^{(1)}$ can be obtained as

$$
\begin{aligned}
\Phi^{(1)}= & \Sigma\left[-y\left(\omega_{\mathrm{j}}-k_{\mathrm{j}}\right) \psi_{\mathrm{nj}}^{(1)}+\omega_{\mathrm{j}} \psi_{\mathrm{ij}+1}^{(1)}+i\left\{y\left(1-2 k_{\mathrm{j}} / \omega_{\mathrm{j}}\right) \psi_{\mathrm{nj}}\right.\right. \\
& -\left(\omega_{\mathrm{j}}^{2}-3 k_{\mathrm{j}}^{2}\right)\left(\omega_{\mathrm{j}}^{2}-k_{\mathrm{j}}^{2}\right)^{-1}\left(\psi_{\mathrm{nj}+1}\right)\left(A_{\mathrm{j} \mathrm{T}}^{(0)} / A_{\mathrm{j}}^{(0)}\right)-\left(y\left(1-2 k_{\mathrm{j}} / \omega_{\mathrm{j}}\right) \psi_{\mathrm{nj}}\right. \\
& \left.\left.-2 k_{\mathrm{j}}^{3} \omega_{\mathrm{j}}^{-1}\left(\omega_{\mathrm{j}}^{2}-k_{\mathrm{j}}^{2}\right)^{-1} \psi_{\mathrm{nj}+1}\right)\left(A_{\mathrm{j} \mathbf{1}}^{(0)} / A_{\mathrm{j}}^{(0)}\right)\right\}+\frac{1}{4}\left(\omega_{\mathrm{j}}^{2}-k_{\mathrm{j}}^{2}\right)^{-1} \\
& \left(y k_{\mathrm{j}} / \omega_{\mathrm{j}}+V_{\mathrm{nj}}^{(1)} / V_{\mathrm{nj}}^{(1)}\right) \psi_{\mathrm{nj}}^{(1)}+\frac{1}{2}\left\{\left(y^{2} \omega_{\mathrm{j}}\left(\omega_{\mathrm{j}}-k_{\mathrm{j}}\right)-\left(\omega_{\mathrm{j}}^{2}-k_{\mathrm{j}}^{2}\right)\right.\right. \\
& \left.\left(1+2 k_{\mathrm{j}} / \omega_{\mathrm{j}}+2 n_{\mathrm{j}}\right)\right) \psi_{\mathrm{nj}}^{2}+y k_{\mathrm{j}}\left(\omega_{\mathrm{j}}+2\right) \psi_{\mathrm{nj}} \psi_{\mathrm{nj}+1}+k_{\mathrm{j}}^{2} \psi_{\mathrm{nj}+1}^{2} \\
& \left.+ \text { c.c. }+C_{3 \mathrm{j}}\right]
\end{aligned}
$$


where primes in (38) and (39) denote derivative with respect to $y$. The first two terms on the r. h. s. of (38) and (39) are equivalent to the first two terms in the expressions for $u^{(1)}$ and $\Phi^{(0)}((20)$ and $(21)$ respectively) when the recurrence relations for the Herinite polynomial are made use of. We observe here that for knowing $V^{(1)}, t^{(1)}$ and $\Phi^{(1)}$ in terms of known quantities, we have to still determine the complex constant $A_{i}^{(1)}$ and real constants $C_{\mathrm{ij}}, i=1,2,3, \ldots$ We shall show in the next section that these constants can be determined from the secularity removal conditions for the equations corresponding to higher order in $\epsilon$. [Following Bogoliubov and Mitropolsky (1961), we may set $A_{j}^{(!)}$and $A_{j}^{(1) *}$ equal to zero. However, as will be seen later, it is not necessiry to do so within the order of approximations considered here].

\section{$2.4 O\left(\epsilon^{2}\right)$ problem: Derivation of non-linear Schrödinger equation}

The $O\left(\epsilon^{2}\right)$ solutions $V^{(2)}, u^{(2)}$ and $\Phi^{(2)}$ can be found out from the $O\left(\epsilon^{2}\right)$ perturbation equations which are obtained by equating the coefficient of $\epsilon^{2}$ equal to zero and are given by

$$
\begin{aligned}
& L v^{(2)}=-L_{\mathrm{i}} v^{(1)}-L_{2} V^{(0)}-P\left(u^{(1)} v_{\mathrm{x} 0}^{(1)}+u^{(1)} v_{\mathrm{x} 0}^{(1)}+u^{(1)} v_{\mathrm{xj}}^{((1))}+v^{(0)} v_{\mathrm{y}}^{(1)}+v^{(1)} v_{\mathrm{y}}^{(0)}\right) \\
& -M\left(u^{(0)} u_{\mathrm{x} 0}^{(1)}+u^{(1)} u_{\mathrm{x} 0}^{(0)} u^{(0)}+u_{\mathrm{x} 1}^{(0)}+v^{(0)} u_{\mathrm{y}}^{(1)}+v^{(1)} u_{\mathrm{y}}^{(0)}\right)+N\left(u^{(0)} \phi_{\mathrm{x} 0}^{(0)}+u^{(1)} \phi_{\mathrm{x} 0}^{(0)}\right. \\
& \left.+u^{(0)} \phi_{\mathrm{x} 1}^{(0)}+v^{(0)} \phi_{\mathrm{y}}^{(1)}+v^{(1)} \phi_{\mathrm{y}}^{(0)}\right)-P_{1}\left(u^{(0)} v_{\mathrm{x} 0}^{(1)}+v^{(0)} v_{\mathrm{y}}^{(0)}\right)-M_{1}\left(u^{(0)} u_{\mathrm{x} 0}^{(0)}\right. \\
& \left.+v^{(0)} u_{\mathrm{y}}^{(0)}\right)+N_{1}\left(u^{(0)} \phi_{\mathrm{x} 0}^{(0)}+v^{(0)} \phi_{\mathrm{y}}^{(0)}\right) \\
& P u^{(2)}-Q v^{(2)}=-\left(P_{1} u^{(1)}-Q_{1} v^{(1)}\right)-\left(P_{2} u^{(0)}-Q_{2} v^{(0)}\right)-\left(u^{(0)} u_{\mathrm{x} 0}^{(1)}+u^{(1)} u_{\mathrm{x} 0}^{(0)}\right. \\
& \left.+u_{\mathrm{x} 1}^{(0)} u^{(0)}+v^{(0)} u_{y}^{(1)}+v^{(1)} u_{\mathrm{y}}^{(0)}\right) \mathrm{T}_{0}-\left(u^{(0)} \Phi_{\mathrm{x} 0}^{(1)}+u^{(1)} \Phi_{\mathrm{x} 1}^{(0)}+u^{(0)} \Phi_{\mathrm{x} 1}^{(0)}\right. \\
& \left.+v^{(0)} \underset{y}{(1)}+v^{(1)} \Phi_{y}^{(0)}\right)_{x 0}-\left(u^{(0)} u_{\mathrm{x} 0}^{(0)}+v^{(0)} u_{\mathrm{y}}^{(0)}\right)_{\mathrm{T} 1}-\left(u^{(0)} u_{\mathrm{x} 0}^{(0)}+v^{(0)} u_{\mathrm{y}}^{(0)}\right)_{\mathrm{T} t} \\
& \text { 一 }\left(u^{(0)} \phi_{\mathrm{x} 0}^{(0)}+v^{(0)} \phi_{y}^{(0)}\right)_{\mathrm{X} 1} \quad \text {, } \\
& \phi_{\mathrm{T} 0}^{(2)}+u_{\mathrm{x} 0}^{(2)}+v_{\mathrm{y}}^{(2)}=-\phi_{\mathrm{T} 1}^{(1)}-u_{\mathrm{x} 1}^{(1)}-\Phi_{\mathrm{T} 2}^{(0)}-u_{\mathrm{x} 2}^{(0)}-u^{(0)} \phi_{\mathrm{x} 0}^{(1)}-u^{(1)} \phi_{\mathrm{x} 0}^{(0)} \\
& -u^{(0)} \Phi_{x l}^{(0)}-{ }^{(0)} \Phi_{\nu}^{(1)}-v^{(1)} \Phi_{y}^{(0)},
\end{aligned}
$$

where $L_{2} \equiv 3 \partial_{\mathrm{TnT} 0 \mathrm{~T} 2}-\partial_{\mathrm{x} 0 \mathrm{x} 0 \mathrm{~T} 2}-\partial_{\mathrm{yyT} 2}+y^{2} \partial_{\mathrm{T} 2}+3 \partial_{\mathrm{T} n \mathrm{~T} 1 \mathrm{~T} 1}-2 \partial_{\mathrm{x} 0 \mathrm{x} \mathrm{xT}_{\mathrm{T}}}$

$$
\begin{aligned}
& -\partial_{\mathrm{x} 2}-2 \partial_{\mathrm{x} 0 \mathrm{~T} 0 \times 2-\partial_{\mathrm{T} 0 \times 1 \times 1} ;} \\
& M_{1} \equiv \partial_{\mathrm{yx} 1}-y \partial_{\mathrm{T} 1} ; \quad N_{1} \equiv \partial_{\mathrm{yT} 1}-y \partial_{\mathrm{x} 1} ; \quad Q_{2} \equiv y \partial_{\mathrm{T} 2}+\partial_{\mathrm{y} \times 2} ; \\
& P_{2} \equiv 2\left(\partial_{\mathrm{T} 0 \mathrm{~T} 2}-\partial_{\mathrm{x} 0 \times 2}\right)+\partial_{\mathrm{T} 1 \mathrm{~T} 1}-\partial_{\mathrm{x} 1 \times 1} .
\end{aligned}
$$


As is obvious from the above equations, the solutions $v^{(2)}, u^{(2)}$ and $\phi^{(2)}$ will now contain additional linear resonant forcings arising from the introduction of higher space and time scales $\left(X_{2} T_{2}\right)$, giving rise to higher harmonics in the fundamental wave. The nonlinear resonant forcings due to the inhomogeneous terms containing the nonlinear products of $O(1)$ and $O(\epsilon)$ quantities, will also produce further higher harmonics produced by the nonlinear terms involving the products of $O(1)$ quantities. Considering the fact that these forcings might give rise to the second and third harmonics in the fundamental wave, we assume the solutions $v^{(2)}, u^{(2)}$ and $\phi^{(2)}$ of the following forms (as was done in the case of $v^{(1)}, u^{(1)}$ and $\left.\phi^{(1)}\right)$ :

$$
\begin{aligned}
& v^{(2)}=\Sigma\left[-i\left((1)_{j}^{2}-k_{j}^{2}\right) \psi_{\pi j}^{(2)}+B_{i j}^{(2)} \exp \left(2 i \theta_{j}\right) V_{i j}^{(2)}+C_{1 j}^{(2)} \exp \left(3 i \theta_{j}\right) V_{2 j}^{(3)}+c \cdot c+d_{1 j}\right] \\
& u^{(2)} \quad \Sigma_{i j}\left[A_{1 j}^{(2)} \exp \left(i \theta_{\mathrm{j}}\right) W_{1 \mathrm{j}}^{(2)}+B_{2 \mathrm{j}}^{(2)} \exp \left(2 i \theta_{\mathrm{j}}\right) W_{2 \mathrm{j}}^{(2)}+C_{2 \mathrm{j}}^{(2)} \exp \left(3 i \theta_{\mathrm{j}}\right) W_{3 \mathrm{j}}^{(2)}+c \cdot c+d_{2 \mathrm{j}}\right] \text {, } \\
& \phi^{(2)}=\Sigma\left[A_{i j}^{(2)} \exp \left(i \theta_{j}\right) Z_{i j}^{(2)}+B_{3 i}^{(2)} \exp \left(2 i \theta_{j}\right) Z_{2 j}^{(2)}+C_{3 j}^{(2)} \exp \left(3 i \theta_{j}\right) Z_{3 j}^{(2)}+c . c+d_{3 j}\right],
\end{aligned}
$$

where $\psi_{\mathrm{nj}}^{(2)}-A_{\mathrm{j}}^{(2)} \exp \left(i 0 j-y^{2} / 2\right) H_{\mathrm{njj}}(y) ; A_{\mathrm{j}}^{(2)}, A_{(1,2) \mathrm{j}}^{(2)}, B_{(1,2,3), \mathrm{i},}^{(2)} C_{(1,2,3) \mathrm{j}}^{(2)}$

are functions of $A_{\mathrm{j}}^{(0)}$ and $A_{\mathrm{j}}^{(0) *}$ and are thus complex constants with respect to $X_{0}, T_{0}$, and $y . \quad d_{\mathrm{ij}}, i=1,2,3$ are real constants with respect to $X_{0}, T_{0}, y$ and functions of $A_{j}^{(0)}$ and $A_{j}^{(0) *}$. These constants are to be determined from the non-secular conditions in higher order in $E$. The functions $V_{(1,2),}^{(2)}, W_{(1,2,3) j}^{(2)}$ and $Z_{(1,2,3), j}^{(2)}$ are functions of $y$.

The solutions $v^{(2)}, u^{(2)}$ and $\Phi \cdot 2^{2}$ in terms of known quantities can be found out in the same manner as the solutions $v^{(1)}, u^{(1)}$ and $\Phi, 3$ were obtained. In order to have the $O\left(\epsilon^{2}\right)$ perturbations completely known, we must determine all the constants and functions just defined above. We are, however, not proposing to do so in this paper. We shall only assume that these $O\left(\epsilon^{2}\right)$ solutions are well-behaved. This means that all the secular terms appearing in the equations governing these solutions, should vanish. The equations governing these solutions will now contain two types of secular terms, (a) secular constant terms and (b) secular resonant texms proportional to $\exp \left( \pm i \theta_{\mathrm{j}}\right)$. Therefore, we will now have two secular-free conditions in order that the $O\left(\epsilon^{2}\right)$ solutions are bounded (well-behaved), one for constant terms and another for resonant terms.

Substitutions of (43), (33), (38), (19), (20) and (21) into (40) which governs the evolution of $v^{(2)}$, yields

$$
\begin{aligned}
& 2 i \omega_{j}\left(4 \omega_{j}^{2}-4 k_{j}^{2}-k_{j} / \omega_{j}-y^{2} \partial_{y y}\right) B_{l j}^{(2)} V_{l j}^{(2)} \exp \left(i 2 \theta_{j}\right)+3 i \omega_{j}\left(9 \omega_{j}^{2}-9 k_{j}^{2}\right. \\
& -k_{j}\left(\omega_{j}-y^{2}+\partial_{y y}\right) \quad C_{1 j}^{(2,} V_{2 i}^{(2)} \exp \left(3 i \theta_{j}\right)+c . c \\
& =\left(y^{2} V_{\mathrm{g}}+1\right) \partial C_{\mathrm{lj}} / \partial_{\mathrm{x} 1}-\left(\omega_{\mathrm{j}}^{2}-k_{\mathrm{j}}^{2}\right)\left(D u_{\mathrm{j}} H_{\mathrm{nj}}\right)\left\{\exp \left(-y^{2} / 2\right)\right\}\left[i\left(A_{\mathrm{j} T 2}^{(0)}+V_{\mathrm{g}} A_{\mathrm{j} \times 2}^{(0)}\right)\right.
\end{aligned}
$$

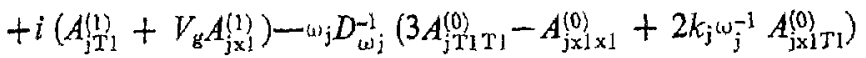




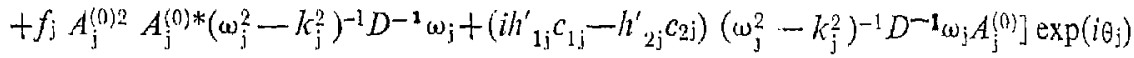

+ terms proportional to $\exp \left(2 i \theta_{\mathbf{j}}\right)$ and $\exp \left(3 i \theta_{j}\right)+c \cdot c$

where $D \omega_{\mathrm{j}}=\partial D / \partial \omega_{\mathrm{j}}=3 \omega_{\mathrm{j}}^{2}-k_{\mathrm{j}}^{2}-2 n_{\mathrm{j}}-1$ and $V_{\mathrm{k}}$ is the group velocity defined by (32) and

$$
\begin{aligned}
& f_{\mathrm{j}}=\left(f_{1 \mathrm{j}}-f_{2 \mathrm{j}}-f_{3 \mathrm{j}}\right) H_{\mathrm{nj}}^{-1} \exp \left(+y^{2} / 2\right) ; h_{1 \mathrm{j}}^{\prime}=h_{1 \mathrm{j}} H_{\mathrm{nj}}^{-1} \exp \left(y^{2} / 2 ; h_{2 \mathrm{j}}^{\prime}=h_{2 \mathrm{j}} H_{\mathrm{nj}}^{-1}\right. \\
& \quad \exp \left(y^{2} / 2\right)
\end{aligned}
$$

with $f_{(1,2,3) \mathrm{j}}$ and $h_{(1,2), \mathrm{j}}$ being functions of $\omega_{\mathrm{j}}, k_{\mathrm{j}}, y$, which are given in the appendix $\mathrm{I}$. Equating the coefficients of $\exp \left( \pm 2 i \theta_{j}\right)$ and $\exp \left( \pm 3 i \theta_{j}\right)$ in both sides of $(46)$, we will get the expressions for $B_{1 j}^{(2)}, V_{1 j}^{(2)}$ and $C_{i j}^{(2)}, V_{2 j}^{(2)}$ while the constant $\left[d_{1 j}\right]$ can be determined from the higher order equations in $€$. This will determine the solution $v^{(2)}$ completely. But, as discussed earlier, we are interested only in knowing under what conditions the solution $v^{(2)}$ remains well behaved (bounded) and this can be done by removing the secular constant and resonant terms appearing in (46). Therefore, we have not presented here the terms proportional to $\exp \left( \pm 2 i \theta_{j}\right)$ and $\exp \left( \pm 3 i \theta_{j}\right)$ in $(46)$.

The condition that the constant terms appearing in (46) be non-secular so as to get secular-free solution $v_{(2)}$, requires that

$$
\left(y^{2} V_{\mathrm{B}}+1\right) \frac{\partial c_{1 \mathrm{j}}}{\partial x_{\mathrm{l}}}=0 \text {. }
$$

As $\left(y^{2} V_{b}+1\right)=0$ gives dependence of $V_{b}$ on $y$ which is not permitted, we have $y^{2} V_{g}+1 \neq 0$. Therefore we must have

$$
\frac{\partial c_{1 j}}{\partial x_{1}}=0, \text { or }\left(\frac{1}{V_{g}} \frac{\partial c_{1 j}}{\partial T_{1}}\right)=0,
$$

[by virtue of (31)]

which means that $C_{1 \mathrm{j}}$ are constant with respect to $X_{1}$ and $T_{1}$. However, $C_{1 \mathrm{j}}$ could be variable in higher order time and space scales. Nevertheless, as far as time scale $T_{1}$ and space scale $x_{1}$ are concerned, $C_{1}$ is a constant which means that to this order the meridional velocity has a constant icomponent. Physically, this is unrealistic because it will mean constant piling up of momentum and vorticity at high latitudes. Therefore, from physical consideration we shall put $C_{1 j}=0$

Similarly, the condition that the resonant terms proportional to $\exp \left( \pm i \theta_{j}\right)$ should be non-secular, requires that

$$
\begin{aligned}
& i\left(A_{\mathrm{j} 2 \mathrm{2} 2}^{(0)}+V_{\mathrm{g}} A_{\mathrm{j \times 2} 2}^{(0)}\right)+\frac{1}{2} \frac{d V_{\mathrm{g}}}{d k_{\mathrm{j}}} A_{\mathrm{j \times 1 \times 1}}^{(0)} \\
& =-\left(\omega_{\mathrm{j}}^{2}-k_{\mathrm{j}}^{2}\right)^{-1} D^{-1} \omega_{\mathrm{j}}\left[A_{\mathrm{j}}^{(0) 2} A_{\mathrm{j}}^{(0) * f_{\mathrm{j}}}+\left(i h^{\prime}{ }_{1 \mathrm{j}} c_{1 \mathrm{j}}-h^{\prime}{ }_{2 \mathrm{j}} c_{2 \mathrm{j}}\right) A^{(0)}\right],
\end{aligned}
$$

together with its complex conjugate relation. In deriving (50) we have made use of the following relations 


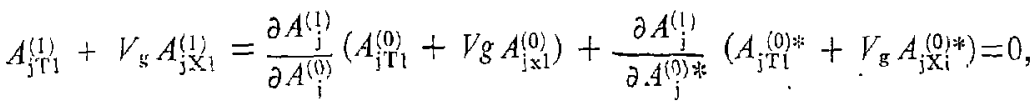

and

$-\frac{\omega \mathrm{j}}{D \omega \mathrm{j}}\left(3 A_{\mathrm{jT} 1 \mathrm{~T} 1}^{(0)}-A_{\mathrm{j} \times 1 \times 1}^{(0)}-2 \frac{k_{1}}{\omega_{\mathrm{j}}} A_{\mathrm{j} \times 1 \mathrm{~T} 1}^{(0)}\right)=\frac{1}{2} \frac{d V_{\mathrm{g}}}{d k_{\mathrm{j}}} A_{\mathrm{j} \times 1 \times 1}^{(0)}$,

where

$\frac{d V_{\mathrm{k}}}{d k_{\mathrm{j}}}=\frac{\partial V_{\mathrm{B}}}{\partial k_{\mathrm{j}}}+V_{\mathrm{g}} \frac{\partial V_{\mathrm{g}}}{\partial \omega_{\mathrm{j}}}=\left[2 \omega_{\mathrm{j}}\left(1+3 V_{\mathrm{g}}^{2}\right)+4 k_{1} V_{\mathrm{g}}\right] / D \omega_{\mathrm{j}}$,

and the fact that $A_{\mathrm{i} T !}^{(0)}+V_{\mathrm{g}} A_{\mathrm{j} \times 1}^{(0)}=0$. Equation (43) and its complex conjugate relation have been made use of.

Note that the equation $(50)$ does not contain the arbitrary constants (with respect to $\left.X_{0}, T_{0}, Y\right) A_{\mathrm{j}}^{(1)}$ and $A_{j}^{(1) * *}$ so that we need not determine them so far as (50) for the time evolution of the wave amplitude $A_{j}^{(0)}$ is concerned. It does contain as yet undetermined constants $C_{2 j}$. It can be shown that the substitution of (43)-(45) into (41) and (42) and then imposition of the condition that the constant terms be secular-frec, still leave the constants $C_{2 j}$ undetermined. However, these constants can be determined from the next higher order equations in $E, i$. $e$. $O\left(\epsilon^{2}\right)$ set of equations.

Proceeding exactly in a manner described in this section, it can be shown that by having the requirement that the constant terms appearing in the set of $O\left(\mathrm{\epsilon}^{3}\right)$ perturbation equations be secular free, one gets the following non-secular conditions for constant terms :

$-\left(y^{2} V_{s}+1 \frac{\partial d^{1} j}{\partial \times 1}=\left[2\left(V_{i^{2}}-1\right) \alpha_{4}+V_{k} \alpha_{2}+\alpha_{3}\right] \frac{\partial^{2}}{\partial x_{1}{ }^{2}}\left(A_{j}^{(0)} A_{j}^{(0) *}\right)\right.$

and

$y^{2} \quad V_{\mathrm{g}} \frac{\partial d_{1 j}}{\partial x_{1}}+y\left(V_{\mathrm{b}}{ }^{2}-1\right) \frac{\partial^{2} c_{2 \mathrm{j}}}{\partial x_{1}{ }^{2}}=\left(V_{\mathrm{k}} \boldsymbol{\alpha}_{2}-\boldsymbol{\alpha}_{3}\right) \frac{\partial^{2}}{\partial x_{1}^{2}}\left(A_{j}^{(0)} A_{j}^{(0) *}\right)$.

The functions $\alpha_{2}, \alpha_{3}$ and $\alpha_{4}$ appearing in (54) and (55) are functions of $\omega \mathrm{j}, k \mathrm{j}$ and $y$ and are deflned in appendix 2 . Eliminating $d_{1 j}$ between (54) and (55), we get

$$
\left(V_{g}^{2}-1\right) \frac{\partial^{2} C_{2 j}}{\partial x_{1}^{2}}=g \frac{\partial^{2}}{\partial x_{1}^{2}}\left(/ A^{(0)} / 2\right)
$$

where

$$
g=\left[V_{g} \alpha_{2}-\alpha_{3}-2 y^{2} V_{g}\left(\alpha_{3}+\alpha_{4} V_{g}^{2}-\alpha_{4}\right)\right] /\left[y\left(y^{2} V_{g}+1\right)\right],
$$

Integrating (56) twice with respect to $X_{1}$, we get

$$
C_{2 \mathrm{j}}=g\left|A^{(0)}\right|^{2} /\left(V_{\mathrm{g}}{ }^{2}-1\right)+\delta_{\mathrm{j}}\left(X_{2}, T_{2}, \ldots\right) X_{1}+\beta_{j}\left(X_{2}, T_{2}, \ldots\right),
$$

where $\delta_{\mathrm{j}}$ and $\beta_{\mathrm{j}}$ are two constants of integation and functions of $X_{2}, T_{2}, \ldots$ Using the relation (31) and its complex conjugate in (56) the second term on r.h.s. of (57) will be replaced by $\delta_{\mathrm{j}} T_{1}$. As these terms give rise to the secularity in $C_{2 j}$ with respect to $X_{1}$ (or $T_{1}$ ) we assume that for secular-free 
$C_{2,}, \delta_{\mathrm{j}}=0$. The function $\beta_{\mathrm{j}}$ has to be evaluated on physical grounds from the appropriate initial or boundary conditions. For example, if we assume that $C_{2 j} \rightarrow 0$ at $\xi=X_{1}-V_{5} T_{1} \rightarrow \pm \infty$ which means that there is no steady part at infinity, we get

$$
\beta_{\mathrm{j}}=-g\left|A_{\mathrm{j} \infty}\right|^{2} /\left(V_{\mathrm{g}}^{2}-1\right) \quad \text { where } A_{\mathrm{j} \infty}=A_{\mathrm{j}}^{(0)}(\xi= \pm \infty),
$$

we note that this solution is valid only for $V_{s}^{2} \neq 1$. This may always be the case for dispersive waves under consideration for which the group velocity is different from the phase velocity.

Substituting for $C_{2 j}$ in (50), we get a nonlinear equation for the evolution of complex amplitude $A_{1}^{(0)}$, that is,

$$
i\left(\frac{\partial A_{\mathrm{j}}^{(0)}}{\partial \mathrm{T} 2}+V_{\mathrm{g}} \frac{\partial A_{\mathrm{j}}^{(0)}}{\partial X^{2}}\right)+M_{\mathrm{j}} \frac{\partial^{2} A_{\mathrm{j}}^{(0)}}{\partial X_{\mathrm{i}}^{2}}=N_{\mathrm{j}}\left|A_{\mathrm{j}}^{(0)}\right| 2 A_{\mathrm{i}}^{(0)}+R_{\mathrm{j}} A_{\mathrm{j}}^{(0)},
$$

together with its complex conjugate relation, where $A_{j}^{(0)}$ now depends upon $\xi, X_{2}, T_{2}, \ldots$, while $R_{\mathrm{j}}$ (through $B_{\mathrm{j}}$ ) depends upon $X_{2}, T_{2}, X_{3}, T_{3}, \ldots$ and is constant with respect to $\xi\left(=\mathrm{X}_{1}-V_{g} T_{1}\right)$ and

$$
\begin{aligned}
& M_{\mathrm{j}}=\frac{d}{\frac{1}{2}} d V_{\mathrm{j}} \\
& R_{\mathrm{j}}=\beta_{\mathrm{i}} h_{2 \mathrm{j}}\left(\omega_{\mathrm{j}}^{2}-k_{\mathrm{j}}^{2}\right)^{-1} D \omega_{\mathrm{j}}^{-1} .
\end{aligned}
$$

The second term in (59) is the dispersion term and the third term is the non linear term. This equation shows that, in a frame of reference moving with the group velocity of the waves, the modulation amplitude $A_{j}^{(0)}$ is determined by the nonlinear interaction $\left(N_{\mathrm{j}}\right.$ term), the dispersive term $\left(M_{\mathrm{j}}\right.$ term) and the linear interaction term $R_{\mathrm{j}}$.

Now, introducing a coordinate transformation such as

$$
\tau=T_{2} ; \quad \zeta-X_{2}-Y_{\mathrm{g}} T_{2}
$$

(59) can be written as

$$
i \frac{\partial A_{\mathrm{j}}^{(0)}}{\partial \tau}+M_{\mathrm{j}} \frac{\partial^{2} A_{\mathrm{j}}^{(0)}}{\partial \xi^{2}}=N_{\mathrm{j}}\left|A_{\mathrm{j}}^{(0)}\right|{ }^{2} A_{\mathrm{j}}^{(0)}+R_{\mathrm{j}} A^{(0)}
$$

where $A_{j}^{(0)}$ is a function of $\xi, \zeta, \tau, X_{3}, T_{3}$ and $R_{\mathrm{j}}$ is that of $\tau, \zeta, X_{3}, T_{3}$. This equation, in a frame of reference moving with the group velocity of the waves, is a nonlinear equation which governs the evolution in slow time and space scales $T_{2}$ and $X_{2}$, of the waves propagating in a barotropic atmosphere with a zero basic state. When we consider the variation of $A_{1}{ }^{(0)}$ with respect to $\tau$ and $\zeta$ (whose order can be regarded as $O(1)$ with respect to (62), then $X_{3}, T_{3}$ become of higher orders and in this sense they can be considered as slow parameters. Therefore, upto second order variation, $A^{(0)}$ can be regarded as a complex function of $\tau$ and $\zeta$, while $R_{\mathrm{j}}$ as a real function of $\beta_{\mathrm{j}}$. If boundary or initial conditions. 
are such that (and hence $R_{J}$ ) is constant with respect to $\tau,(62)$ is called the "modified nonlinear Schrödinger equation", because of the linear terms appearing on the right hand side of (62) as a modification to the usual nonlinear Schrödinger equation. Whether $R_{\mathrm{j}}$ is a constant or varies with $\tau$, the linear interaction $R_{\mathrm{j}} A_{\mathrm{j}}$ term in (62) is not very important because it causes only a phase shift in the solution $A_{j}$. Making a transformation such that

$$
A_{\mathrm{j}}^{(0)}=\widetilde{A}_{\mathrm{i}}^{(0)}(\zeta, \tau) \exp \left[-\mathrm{i} \int^{\tau} R\left(\tau^{\prime}\right) d \tau^{\prime}\right],
$$

the $R_{\mathrm{j}} A_{\mathrm{j}}(0)$ term can be formally eliminated from (62). With the transformation (63), (62) can be rewritten as

$$
i \frac{\partial \hat{A}_{j}^{(0)}}{\partial T}+M_{\mathrm{j}} \frac{\partial^{2} A_{\mathrm{j}}^{(0)}}{\partial \xi^{2}}=N_{\mathrm{j}}\left|\vec{A}_{\mathrm{j}}^{(0)}\right|{ }^{2} \bar{A}_{\mathrm{j}}^{(0)}
$$

It should be noted that the modified nonlinear Schrodinger equation (64) describing amplitude modulation of the wave trains represented by (19), (20) and (21), has been derived from the non-secular conditions (boundness condition for the wave solutions) for $O\left(\varepsilon^{2}\right)$ perturbation equations and therefore, is valid for scales as large as $O\left(\epsilon^{-2}\right)$.

\section{Solutions of modified nonlinear schrodinger equation (64)}

If now, we look for a plane wave solution to (64) for the amplitude $\breve{A}^{3}{ }^{(0)}$, of the form

$$
\widetilde{A}_{j}^{(0)} \rightarrow \bar{A}_{\mathrm{i}}^{(0)} \exp \left[i\left(\widetilde{K}_{\mathrm{j}} \xi-\widehat{\Omega}_{\mathrm{j}} \tau\right],\right.
$$

we get following nonlinear dispersion relation

$$
\widetilde{\Omega_{\mathrm{j}}}=M_{\mathrm{j}}{\widetilde{K_{\mathrm{j}}}}^{2}+N_{\mathrm{j}}\left|\tilde{A}_{\mathrm{j}}^{(0)}\right|^{2},
$$

where $\widetilde{K}_{\mathrm{j}}$ and $\widetilde{\Omega}_{\mathrm{j}}$ are respectively the wave number and frequency of the envelope of the wave trains represented by (19), (20) and (21).

\subsection{Stationary solitary wave solution}

Let us look for a localized solution of (64) for $\widetilde{A}_{j}^{(0)}$ satisfying the boundary conditions; (a) $\left|\bar{A}_{j}^{(0)}\right|^{2}$ is bounded between two limits $A_{\max }$ and $A_{\min }$. (b) at $\left|\breve{A}_{\mathrm{j}}^{(0)}\right|^{2}=A_{\max }$, an extremum i.e. $\delta\left|\breve{A}_{j}^{(0)}\right|^{2} / \delta \xi=0$ but $\delta^{2} \mid \widetilde{A}_{\mathrm{j}}^{(0)}, 2 / \partial \xi^{2} \neq \neq 0$ and (c) $A_{\min }$ is the asymptotic value of $\left|\vec{A}_{j}^{(0)}\right|^{2}$ at $\xi \rightarrow \pm \infty$. introducing two real variables $\overline{A_{j}}$ and $\theta$ which represent the real and imaginary parts of $\tilde{A}_{\mathrm{j}}^{(0)}$ we can write $\tilde{A}_{\mathrm{J}}^{(0)}$ as

$$
\vec{A}_{\mathrm{j}}^{(0)}(\xi, \tau)=\sqrt{\bar{A} \mathrm{j}(\xi, \tau)} \exp \left\{i \theta_{\mathrm{j}}(\xi, \zeta) / 2 M^{\mathrm{j}}\right\}
$$

Substituting (67) into (64) and separating real and imaginary parts, we get after simplification. 


$$
\begin{aligned}
& \frac{\partial A_{\mathrm{j}}}{\partial \tau}+\frac{\delta}{\delta \xi}\left(\bar{A} \frac{\partial}{\partial \xi}=0,\right. \\
& \frac{\lambda \theta}{\partial \tau}+\frac{1}{2}\left(-\frac{\partial \theta}{\partial \xi}\right)^{2}=-2 M_{\mathrm{j}} N_{\mathrm{j}} \bar{A}_{\mathrm{j}}+\frac{1}{2} M_{\mathrm{j}}{ }^{2} \frac{\partial}{\partial A_{\mathrm{j}}} \quad\left\{\bar{A}_{\mathrm{j}}^{-1}\left(\frac{\partial \bar{A}_{\mathrm{j}}}{\partial \zeta}\right)^{2}\right\}
\end{aligned}
$$

It can be seen from (68) and (69) that $M_{\mathrm{j}}=0$, the envelope will steepen in the course of time evolution and finally break down. On the other hand, if $M_{\mathrm{j}} \neq 0$, the dispersive term

$$
\left[{ }^{1} M_{\mathrm{j}}^{2} \frac{\partial}{\partial \vec{A}}\left\{\bar{A}_{\mathrm{j}}^{-1}\left(\frac{\partial A_{\mathrm{j}}}{\partial \dot{\xi}}\right)^{2}\right\}\right]
$$

begins to play a role in checking the steepening of the envelope. Therefore, one may expect an equilibrium stationary "state resulting from the balance between nonlinear steepening and the dispersive effects. Following Hasegawa (1975), the stationary solution (i.e. when $\partial / \partial \tau \cdots 0$ ) to the modified nonlinear Schrodinger equation (64) for the case when $M_{\mathrm{j}} N_{\mathrm{j}}<0$ and subject to the boundray conditions (a), (b), (c), mentioned above, can be written as

$$
A^{(0)}(\xi, \tau)=\triangle_{\mathrm{j}} \operatorname{sech}\left(\xi / W_{\mathrm{j}}\right) \exp \left\{i\left(\bar{\Omega}_{\mathrm{j}} / 2 M_{\mathrm{j}}\right) \tau\right\}
$$

where

$$
\Delta_{j}=A_{\text {max }}^{+1 / 2}=\sqrt{-\overline{\Omega_{\mathrm{j}}} / M_{\mathrm{j}} N_{\mathrm{j}}}, W_{\mathrm{j}}=\left(-2 M / N_{\mathrm{j}}\right)^{1 / 2} / \Delta_{\mathrm{j}} \text { and } \Omega_{\mathrm{j}}>0
$$

and is a constant. This solution has structure similar to "the "soliton "s soluton" for weakly nonlinear waves in a dispersive medium and hence, is called "envelope soliton" which moves at a speed equal to $V_{g}$ i.e. group velocity of the waves. It is seen that the amplitude of envelope soliton $\triangle_{i}$ (also called the height) is inversely proportional to its width $W_{\mathrm{j}}$. As is obvious, the constant parameter $\bar{\Omega}_{\mathrm{j}}$ in (70) is a phase factor and will thus determine the phase of envelope soliton. When $M_{\mathrm{j}} N_{\mathrm{j}}>0$, the solution of (64) has a "envelope hole" structure and has not been discussed here for its lack of relevance in geophysical fluid dynamics.

\section{Results and discussions}

The various asymptotic behaviours of the atmospheric waves have been discussed, using multiple time and space scales and the derivative expansion method. This method has, as pointed out by Kawahara (1973), an advantage over the other methods in the sense that the dependence of the parameters on the independent variables need not be specified $a$ priori and also that the perturbation analysis to the different orders could be carried out systematically. Based upon the divergent barotropic model on a $\beta$-plane, we have investigated the long time evolution of finite amplitude (i.e. weakly nonlinear) and slowly varying wave trains propagating in the atmosphere with a zero basic zonal flow. The present analysis is basically an extension of the linear stability analysis of the atmospheric wave motions in the equatoral region (Matsuno 1966) into the nonlinear regines for these waves. 
In the lowest order approximations of our model, i.e. to the $O(1)$, the results of the linear theory of Matsuno (1966) are retrieved. The propagation characteristics of the linearized solutions for these atmospheric waves are such that there exist three distinct classes of planetary scale waves for the meridional modes $n_{j} \geqslant 1$. The first two types are the eastward and westward propagating inertialgravity waves while the third is the westward propagating Rossby waves. They are distinguished from each other by the fact that the frequencies of the inertial-gravity waves are much larger than that of Rossby waves (figure 2). For the particular lowest meridional mode $n_{\mathrm{j}}=0$, the existence of only two types of waves is possible; one is eastward "propagating inertial-gravity waves and the other describes the westward propagating mixed Rossby-gravity or 'Yanai waves' (figure 2). The dispersion relation for the latter type resembles that of the Rossby waves for the wavelength much smaller than the meridional extent. For the case where the wavelength is longer than the meridional extent, however, the frequency approaches that of the gravity waves of the same wavelength. In other words, the mixed Rossby-gravity wave connects two families of waves and its frequency ranges from a value which is comparable to the frequency of the inertial-gravity waves to a value which is close to that of the Rossby waves.

Using multiple time scale method to solve the weakly nonlinear equations, it is shown that the evolution of the wave amplitude on slowly varying time and space scales is governed by the modified nonlinear Schrodinger equation. The stationary solutions to the modified nonlinear Schrodinger equation are obtained and found to have the structure of "envelope solitary waves (envelope solitons or wave packets)' moving at a speed equal to the group velocity of waves, which results from the balance between the nonlinear steepening and the dispersive effects. We consider this result as an indication of the possible existence of the envelope solitary waves in the atmosphere. Though the dispersive waves in optics and plasmas are known to have envelope soliton solutions, this is, to our knowledge a very good theoretical evidence of the existence of 'envelope solitons' in a geophysical situation.

The methodology presented in $\$ 2$ for deriving (59) [or (62)] governing the the evolution of the amplitude is quite general in the sense that this equation governs the amplitude evolution for all the waves described by the Shallow Water Equations. However, the envelope soliton solution presented in $\S 3$ is valid only for the dispersive waves. For example, Rossby waves and Rossbygravity waves will have such nonlinear solution. On the other hand the Kelvin waves will not have soliton solution. We note that for the Kelvin waves $\omega_{\mathrm{j}}=-k_{\mathrm{j}}$ and $n_{\mathrm{j}}=-1$. Therefore, the group velocity $V_{\mathrm{g}}$ for the Kelvin waves given by (32) is a constant. Thus $\mathrm{M}_{\mathrm{j}}$ in (62) is given by $M_{\mathrm{j}}=\frac{1}{2} \frac{d V_{\mathrm{g}}}{d k_{\mathrm{j}}}=0$ [see (60)]. In the absence of the dispersive term containing $M_{\mathrm{j}},(62)$ represents steepening of the amplitude of the Kelvin waves in the nonlinear regime.

We note here that the modified nonlinear Schrodinger equation describing the dependence of the wave amplitude on slower time and space scales $\left(T_{2}\right.$ and $X_{2}$ ), has been derived from the non-secular conditions (boundedness conditions for the wave solutions) for $O\left(\epsilon^{2}\right)$ perturbation equations and therefore, is valid for scales as large as $O\left(\epsilon^{-2}\right)$. Although the dependence of the wave amplitude on 
$O\left(\epsilon^{3}\right)$ and higher order scales should be determined up to this order of perturbations we have however not considered in the model the variation in the change of the wave amplitude upto $O\left(\epsilon^{3}\right)$ and higher order scales which may be considered in a sense as a kind of slow parameters since $\epsilon \ll 1$. In other words, the evolution of the wave amplitude in the present analysis is accurate up to $O\left(\epsilon^{2}\right)$ scales and will have an error of $O\left(\epsilon^{3}\right)$.

\section{Conclusions}

In conclusion, our model predicts that the long time evolution of finite-amplitude waves generared in a barotropic single layer model atmosphere on a $\beta$-plane can be described by a modified nonlinear Schrodinger equation. The model further predicts that these finite-amplitude waves finally evolve in time in the shape of envelope solitary waves or envelope solitons (i.e. in the shape of wave packets) and this provides us theoretical evidence for the existence of 'envelope solitons' in a barotropic atmosphere, in particular and in any geophysical situation, in general. These envelope solitons propagate with the group velocity of the wave.

\section{Acknowledgements}

The authors are grateful to Mr M Mohan of Physical Research Laboratory, Ahmedabad for many valuable discussions and constructive criticisms.

Appendix 1. Expressions for $h_{(1,2) \mathrm{j}}$ and $f_{\mathrm{jj}}, i=1,2,3$ appearing in (47).

$$
\begin{aligned}
h_{1 j}= & \left(\omega_{\mathrm{j}}^{2}-k_{\mathrm{j}}^{2}\right)^{2}\left(y H_{\mathrm{nj}}-H_{\mathrm{nj}+1}\right) \exp -\left(y^{2} / 2\right)+\left(y \omega_{\mathrm{j}}+k_{\mathrm{j}} \partial / \partial y\right) \\
& {\left[\exp \left(-y^{2} / 2\right)\left\{\left(y^{2}\left(\omega_{\mathrm{j}}-k_{\mathrm{j}}\right)+\omega_{\mathrm{j}}+\left(2 n_{\mathrm{j}}+1\right) k_{\mathrm{j}}\right) H_{\mathrm{nj}}-y \omega_{\mathrm{j}} H_{\mathrm{nj}+1}\right\}\right] } \\
- & \left(y k_{\mathrm{j}}+\omega_{\mathrm{j}} \partial / \partial y\left(\left[\operatorname { e x p } ( - y ^ { 2 } / 2 ) \left\{\left(y^{2}\left(\omega_{\mathrm{j}}-k_{\mathrm{j}}\right)-k_{\mathrm{j}}-\omega_{\mathrm{j}}(2 n \mathrm{j}+1)\right) H_{\mathrm{nj}}\right.\right.\right.\right. \\
+ & \left.\left.y k_{\mathrm{j}} H_{\mathrm{nj}+1}\right\}\right], \\
h_{2 \mathrm{j}}= & k \mathrm{j}\left(\omega_{\mathrm{j}}^{2}-k_{\mathrm{j}}^{2}\right)^{2} H_{\mathrm{nj}} \exp \left(-\mathrm{j}^{2} / 2\right)+k_{\mathrm{j}}\left(y \omega_{\mathrm{j}}+k_{\mathrm{j}} \partial / \partial y\right)_{\mathrm{v}}^{\prime}\left[\exp \left(-y^{2} / 2\right)\right. \\
& \left.\left\{y\left(\omega_{\mathrm{j}}-k_{\mathrm{j}}\right) H_{\mathrm{nj}}+k^{\mathrm{j}} H_{\mathrm{nj}+1}\right\}\right]-k_{\mathrm{i}}\left(y k_{\mathrm{j}}+\omega \partial / \partial y\right)\left[\operatorname { e x p } ( - y ^ { 2 } / 2 ) \left\{y\left(\omega_{\mathrm{j}}-k_{\mathrm{j}}\right)\right.\right. \\
& \left.\left.H_{\mathrm{nj}}-\omega_{\mathrm{j}} H_{\mathrm{nj}+1}\right\}\right], \\
f_{\mathrm{ij}}= & \frac{1}{2}\left(\omega_{\mathrm{j}}^{2}-k_{\mathrm{j}}^{2}\right)\left[\left\{y\left({ }_{2}^{3} k_{\mathrm{j}}+\omega_{\mathrm{j}}-1\left(\omega_{\mathrm{j}}^{2}-3 k_{\mathrm{j}}^{2}\right)\right) V_{\mathrm{nj}}^{(1)}+\left(2 \omega_{\mathrm{j}}^{2}-3 k_{\mathrm{j}}^{2}\right) \cdot V_{\mathrm{nj}}^{(1) \prime} / 2 \omega_{\mathrm{j}}\right\}\right. \\
& H_{\mathrm{nj}} \exp \left(-y^{2} / 2\right)-\left(\omega_{\mathrm{j}}-3 k_{\mathrm{j}}^{2} / \omega_{\mathrm{j}}\right) V_{\mathrm{nj}}^{(1)} H_{\mathrm{nj}+1} \exp \left(-y^{2} / 2\right)+k_{\mathrm{j}}\left(\omega_{\mathrm{j}}^{2}-k_{\mathrm{j}}^{2}\right) \\
& \left.H_{\mathrm{nj}} \exp \left(-{ }^{3} y^{2}\right)\left\{\left(y^{2} \omega_{\mathrm{j}}\left(\omega_{\mathrm{j}}-k_{\mathrm{j}}\right)+\omega_{\mathrm{j}}^{2}-k_{\mathrm{j}}^{2}\right) H_{\mathrm{nj}}^{2}-y \omega_{\mathrm{j}}^{2} H_{\mathrm{nj}} H_{\mathrm{nj}+1}\right\}\right], \quad(\mathrm{A} 2 \mathrm{a})
\end{aligned}
$$




$$
\begin{aligned}
& f_{2 j}=\left(y \omega_{j}+k_{j} \partial / \partial y\right)\left[\operatorname { e x p } ( - y ^ { 2 } / 2 ) \left\{k _ { j } ( y ( \omega _ { j } - k _ { j } ) H _ { n j } + k _ { j } H _ { n j + 1 } ) \left(1\left(\omega_{j}^{2}-k_{j}^{2}\right)^{-1}\right.\right.\right. \\
& \left(y V_{\mathrm{nj}}^{(1)}+V_{\mathrm{nj}}^{(1)} \cdot k_{\mathrm{j}} / \omega_{\mathrm{j}}\right)+\left(y^{2} \omega_{\mathrm{j}}\left(\omega_{\mathrm{j}}-k_{\mathrm{j}}\right)+\omega_{\mathrm{j}}^{2}-k_{\mathrm{i}}^{2}\right) H_{\mathrm{nj}}^{2} \exp \left(-y^{2}\right)-y \omega_{\mathrm{j}}^{2} H_{\mathrm{n}} \\
& \left.\times H_{n j+1} \exp \left(-y^{2}\right)\right)+{ }_{4}^{1} H_{\mathrm{nj}}\left(V_{\mathrm{nj}}^{(1)}+y V_{\mathrm{nj}}^{(1)^{\prime}}+k_{\mathrm{j}} V_{\mathrm{nj}}^{\left(1 \mu^{\prime \prime}\right.} / \omega_{\mathrm{j}}-4\left(\omega_{\mathrm{j}}^{2}-k_{\mathrm{j}}^{2}\right)\right. \\
& \left\{\left(y^{2} \omega_{j}\left(\omega_{j}-k_{j}\right)+\omega_{j}^{2}+\omega_{j}\left(\omega_{j}-k_{j}\right)-n_{j} \omega_{j}^{2}-k_{j}^{2}\right) y H_{n j}^{2}-\left(y^{2} \omega_{j}\left(\omega_{j}-k_{j}\right)\right.\right. \\
& \left.\left.\left.+\frac{1}{2} \omega_{j}^{2}-k_{j}^{2}\right) H_{\mathrm{nj}} U_{\mathrm{nj}+1}+\frac{1}{2} y \omega_{j}^{2} H_{\mathrm{nj}+1}\right\} \exp \left(-y^{2}\right)\right)+\frac{1}{2} \omega_{\mathrm{j}}^{-1} V_{\mathrm{nj}}^{(1)}\left(H _ { \mathrm { nj } } \left[y ^ { 2 } \left(\omega_{j}\right.\right.\right. \\
& \left.\left.\left.\left.\left.-k_{\mathrm{j}}\right)+\omega_{\mathrm{j}}+\left(2 n_{\mathrm{j}}+1\right) k_{\mathrm{j}}\right]-y \omega_{\mathrm{j}} H_{\mathrm{nj}+1}\right)\right\}\right] \text {, } \\
& f_{3 \mathrm{j}}=k_{\mathrm{j}}\left(y k_{\mathrm{j}}+\omega \partial / \partial y\right)\left[\exp -y^{2} / 2\left\{\frac{1}{4}\left(\omega_{\mathrm{j}}^{2}-k_{\mathrm{j}}^{2}\right)^{-1}\left[y\left(\omega_{\mathrm{i}}-k_{\mathrm{j}}\right) H_{\mathrm{nj}}-\omega_{\mathrm{j}} H_{\mathrm{nj}+1}\right]\right.\right. \\
& \left(y V_{\mathrm{nj}}^{(1)}+k_{\mathrm{j}} Y_{\mathrm{nj}}^{(1 / j} / \omega_{\mathrm{j}}\right)+\frac{1}{2}\left(\omega_{\mathrm{j}}^{2}-k_{\mathrm{j}}^{2}\right)\left[y\left(\omega_{\mathrm{j}}-k_{\mathrm{j}}\right) H_{\mathrm{nj}}+k_{\mathrm{j}} H_{\mathrm{ni}+1}\right]\left(k_{\mathrm{j}} y V_{\mathrm{nj}}^{(1)} / \omega_{\mathrm{j}}\right. \\
& \left.+V_{n j}^{(1)}\right)-V_{n j}^{(1)}\left\{\left(y^{2}\left(\omega_{j}-k_{j}\right)-k_{j}+\left(2 n_{j+1}\right) \omega_{j}\right) H_{n j}+y k_{j} H_{n i+1}\right\} / 2 \omega_{j} k_{j} \\
& +H_{\mathrm{nj}}\left(V_{\mathrm{nj}}^{(1)}+y V_{\mathrm{nj}}^{(1)}+\omega \mathrm{ji} V_{\mathrm{nj}}^{(1)}\left(k_{\mathrm{j}}\right) / 4 \omega_{\mathrm{j}}+\exp \left(-y^{2}\right)\left(\left\{y\left(\omega_{\mathrm{i}}-k_{\mathrm{j}}\right) H_{\mathrm{nj}}+k_{\mathrm{j}}\right.\right.\right. \\
& \left.H_{\mathrm{nj}+1}\right\}\left\{\left(y^{2}\left(\omega_{\mathrm{j}}-k_{\mathrm{j}}\right) \omega_{\mathrm{j}}+\left(\omega_{\mathrm{j}}^{2}-k_{\mathrm{j}}^{2}\right)\left(1+2 k_{\mathrm{j}}\left(\omega_{\mathrm{j}}\right)+2 n_{\mathrm{j}}\left(\omega_{\mathrm{j}}^{2}-k_{\mathrm{j}}^{2}\right)\right)\right.\right. \\
& \left.H_{\mathrm{pj}}^{2}+y k_{\mathrm{j}}\left(\omega_{\mathrm{j}}+2\right) H_{\mathrm{nj}} H_{\mathrm{nj}+1}+k_{\mathrm{j}}^{2} H_{\mathrm{nj}+1}\right\}-\frac{1}{2}\left\{y\left(\omega_{\mathrm{j}}-k_{\mathrm{j}}\right) H_{\mathrm{nj}}-\omega_{\mathrm{j}}\right. \\
& \left.H_{\mathrm{nj}+1}\right\}\left\{\left(y^{2}\left(\omega_{\mathrm{j}}-k_{\mathrm{j}}\right) \omega_{\mathrm{j}}+\omega_{\mathrm{j}}^{2}-k_{\mathrm{j}}^{2}\right) H_{\mathrm{nj}}^{2}-y \omega_{\mathrm{j}}^{2} H_{\mathrm{nj}} H_{\mathrm{nj}+1}\right\}-\frac{1}{2} H_{\mathrm{nj}}\left(\omega_{\mathrm{j}}^{2}\right. \\
& \left.-k_{j}^{2}\right)\left[y \left(\omega_{j}\left(\omega_{j}-k_{j}\right)\left(y^{2}+1\right)+\omega_{j}^{-1}\left(\omega_{j}^{2}-k_{j}^{2}\right)\left(\omega_{j}+2 k_{j}\right)-2 k_{j}^{2}+k_{j}\right.\right. \\
& \left.\left(n_{\mathrm{j}}+1\right)\left(\omega_{\mathrm{j}}+2\right)+2 n_{\mathrm{j}}\left(\omega_{\mathrm{j}}^{2}-k_{\mathrm{j}}^{2}\right)\right) H_{\mathrm{nj}}^{2}-y k_{\mathrm{j}}\left(1+k_{\mathrm{j}}+\omega_{\mathrm{j}} / 2\right) H_{\mathrm{nj}+1}^{2} \\
& -\left(y^{2} \omega_{j}\left(\omega_{j}-k_{j}\right)+\left(\omega_{j}^{2}-k_{j}^{2}\right)\left(\omega_{j}+2 k_{j}\right) / \omega_{j}-2 k_{j}^{2}-k_{j}\left(1+\omega_{j} / 2\right)\right. \\
& \left.\left.\left.\left.\left.+2 n_{\mathrm{j}}\left(\omega_{\mathrm{j}}^{2}-2 k_{\mathrm{j}}^{2}\right)\right) H_{\mathrm{nj}} H_{\mathrm{nj}+1}\right]\right)\right\}\right] \text {, }
\end{aligned}
$$

where a prime denotes the derivative with respect to $Y$.

Appendix 2. Expressions for $\alpha_{2}, \alpha_{3}$ and $\alpha_{4}$ appearing in (54) and (55).

$$
\begin{aligned}
\alpha_{2}= & y \exp \left(-y^{2}\right)\left[\left(y\left(\omega_{\mathrm{j}}-k_{\mathrm{j}}\right) H_{\mathrm{nj}}+k_{\mathrm{j}} H_{\mathrm{nj}+1}\right)^{2}-\left\{\left(V_{\mathrm{g}}+1\right)\left(y^{2}+1\right)\right.\right. \\
& \left.\left(\omega_{\mathrm{j}}^{2}-k_{\mathrm{j}}^{2}\right)-2\left(n_{\mathrm{j}}+1\right)\left(2 \omega_{\mathrm{j}} k_{\mathrm{j}} V_{\mathrm{g}}-\omega_{\mathrm{j}}^{2}-k_{\mathrm{j}}^{2}\right)\right\} H_{\mathrm{nj}}^{2}+y\left\{\left(V_{\mathrm{g}}+1\right)\right. \\
& \left.\left.\left(\omega_{\mathrm{j}}^{2}-k_{\mathrm{j}}^{2}\right)-2 \omega_{\mathrm{j}} k_{\mathrm{j}} V_{\mathrm{g}}+\omega_{\mathrm{j}}^{2}+k_{\mathrm{j}}^{2}\right\} H_{\mathrm{nj}} H_{\mathrm{nj}+1}\right], \\
\boldsymbol{\alpha}_{3}= & y_{\mathrm{j}} \exp \left(-y^{2}\right)\left[\left\{y k_{\mathrm{j}}\left(V_{\mathrm{g}}+1\right)\left(1-2 k_{\mathrm{j}} / \omega_{\mathrm{j}}\right) H_{\mathrm{nj}}-k_{\mathrm{j}} \omega_{\mathrm{j}}^{-1}\left(\omega_{\mathrm{j}}^{2}-k_{\mathrm{j}}^{2}\right)^{-1}\right.\right. \\
& {\left.\left[\omega_{\mathrm{j}}\left(\omega_{\mathrm{j}}^{2}-3 k_{\mathrm{j}}^{2}\right) V_{\mathrm{g}}+2 k_{\mathrm{j}}^{3}\right] H_{\mathrm{nj}+1}\right\}\left\{y\left(\omega_{\mathrm{j}}-k_{\mathrm{j}}\right) H_{\mathrm{nj}}+k_{\mathrm{j}} H_{\mathrm{nj}+1}\right\}+\left\{y k_{\mathrm{j}}\right.} \\
& \left.\left(V_{\mathrm{b}}+1\right) H_{\mathrm{nj}}-k_{\mathrm{j}}\left(\omega_{\mathrm{j}}^{2}-k_{\mathrm{j}}\right)^{-1}\left(2 \omega_{\mathrm{j}} k_{\mathrm{j}} V_{\mathrm{g}}-\omega_{\mathrm{j}}^{2}-k_{\mathrm{j}}^{2}\right) H_{\mathrm{nj}+1}\right\}\left\{y\left(\omega_{\mathrm{j}}-k_{\mathrm{j}}\right)\right. \\
& \left.H_{\mathrm{nj}}-\omega_{\mathrm{j}} H_{\mathrm{nj}+1}\right\}+\left(\omega_{\mathrm{j}}^{2}-k_{\mathrm{j}}^{2}\right) H_{\mathrm{nj}}\left\{\left(\left(V_{\mathrm{g}}+1\right)\left(y^{2}+1\right)\right.\right. \\
& \left.\left(\omega_{\mathrm{j}}-2 k_{\mathrm{j}}\right) / \omega_{\mathrm{j}}-2 \omega_{\mathrm{j}}^{-1}\left(\omega_{\mathrm{j}}^{2}-k_{\mathrm{j}}^{2}\right)^{-1}\left(n_{\mathrm{j}}+1\right)\left[\omega_{\mathrm{j}} V_{\mathrm{q}}\left(\omega_{\mathrm{j}}^{2}-3 k_{\mathrm{j}}^{2}\right)+2 k_{\mathrm{j}}^{3}\right]\right)
\end{aligned}
$$




$$
\begin{aligned}
& H_{\mathrm{nj}}-y\left(\left(V_{\mathrm{g}}+1\right)\left(\omega_{\mathrm{j}}-2 k_{\mathrm{j}}\right) / \omega_{\mathrm{j}}-\omega_{\mathrm{j}}^{-1}\left(\omega_{\mathrm{j}}^{2}-k_{\mathrm{j}}^{2}\right)^{-1}\left[\omega_{\mathrm{j}} V_{\mathrm{g}}\left(\omega_{\mathrm{j}}^{2}-3 k_{\mathrm{j}}^{2}\right)\right.\right. \\
+ & \left.\left.\left.\left.2 k_{\mathrm{j}}^{2}\right]\right) H_{\mathrm{nj}+1}\right\}-\left(\omega_{\mathrm{j}}^{2}-k_{\mathrm{j}}^{2}\right)\left(y^{2} H_{\mathrm{nj}}^{2}-y H_{\mathrm{nj}} H_{\mathrm{nj}+1}\right)+\omega_{\mathrm{j}} k_{\mathrm{j}} H_{\mathrm{nj}+1}^{2}\right], \\
\alpha_{4}= & 2\left(\omega_{\mathrm{j}}^{2}-k_{\mathrm{j}}^{2}\right) H_{\mathrm{nj}} \exp \left(-y^{2}\right)\left\{y\left(\omega_{\mathrm{j}}^{2}-2 k_{\mathrm{j}}^{2}+\omega_{\mathrm{j}} k_{\mathrm{j}}\right) H_{\mathrm{nj}}-\left(\omega_{\mathrm{j}}^{2}-2 k_{\mathrm{j}}^{2}\right) H_{\mathrm{nj}+1}\right\} .
\end{aligned}
$$

\section{References}

Benney D J 1966 J. Math. Phys. 4552

Bogoliubov N N and Mitropolsky Y A 1961 Asymoptotic methods in theory of nonlinear oscillations [Eng. Trans.] (Delhi : Hindustan Publishing Corporation) p. 51

Boyd J P 1980 J. Phys. \{Oceanogr. 101

Clarke R A 1971 Geophys. Fluid Dyn. 2 343-354

Domaracki Al and Loesch A Z 1977 J. Atmos. Sci. 34486

Duffy D G 1974 J. Atmos. Sci. 311218

Gill A E 1974 Geophys. Fluid Dyn. 6 29-47

Hasegawa A 1975 Plasma instabilities and nonlinear effects - Physics and Chemistry in Space (eds) J G Roederer and J T Wasson (Berlin Heidelberg, New York: Spronger Verlag) Vol. 8 p. 196

Kawahara T 1973 J. Phys. Soc. Jpn. 351537

Larsen L H 1965 J. Atmos. Sci. 22222

Loesch A Z 1978 J. Atmos. Sci. 35929

Long R 1964 J. Atmos. Sci. 21 1971

Longuet-Higgins M S and Gill A E 1967 Proc.R. Soc. London A299 120

Lorenz E N 1972 J. Atmos. Sci. 29259

Margenau H and Murphy G M 1966 The mathematics of physics and chemistry - Second east-west reprint (New Delhi : Affiliated East-West Press)

Matsuno T 1966 J. Meteorol. Soc. Jpn. 4425

Pedlosky J 1970 J. Atmos. Sci. 2715

Pedlosky J 1971 Geophys. Fluid Dyn. Lectures in Appl. Math. (Providence, R. I. : American Math. Soc.) 13 1-60.

Pedlosky J 1972a J. Atmos. Sci. 2953

Pedlosky J 1972b J. Atmos. Sci. 29680

Pedlosky J 1977 J Atmos. Sci. 341898

Phillips N A 1954 Tellus 6273

Redekopp L G 1977 J. Fluid Mech. 82725

Redekopp L G and Weidman P D 1978 J. Atmos. Sci. 35790

Rossby C G et al 1939 J. Mar. Res. 239

Scott A G, Chu F Y F and McLaughlin D W 1973 Proc. IEEE 611443 